$\mathrm{DCPT} / 07 / 80$

$\operatorname{IPPP} / 07 / 40$

arXiv:0708.3052 [hep-ph]

\title{
Studying the MSSM Higgs sector by forward proton tagging at the $\mathrm{LHC}$
}

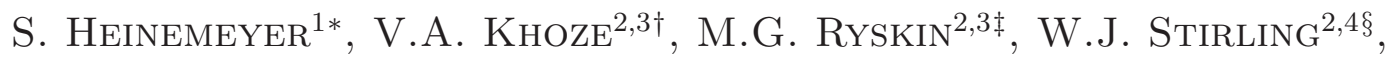 \\ M. TASEVSKY, \\ ${ }^{1}$ Instituto de Fisica de Cantabria (CSIC-UC), Santander, Spain \\ ${ }^{2}$ IPPP, Department of Physics, Durham University, Durham DH1 3LE, U.K. \\ ${ }^{3}$ Petersburg Nuclear Physics Institute, Gatchina, St. Petersburg, 188300, Russia \\ ${ }^{4}$ Department of Mathematical Sciences, Durham University, DH1 3LE, U.K. \\ ${ }^{5}$ Institute of Physics, 18221 Prague 8, Czech Republic \\ ${ }^{6}$ University of Antwerpen, Physics Department, B-2610 Antwerpen, Belgiun***
}

\begin{abstract}
We show that the use of forward proton detectors at the LHC installed at $220 \mathrm{~m}$ and $420 \mathrm{~m}$ distance around ATLAS and / or CMS can provide important information on the Higgs sector of the MSSM. We analyse central exclusive production of the neutral $\mathcal{C P}$-even Higgs bosons $h$ and $H$ and their decays into bottom quarks, $\tau$ leptons and $W$ bosons in different MSSM benchmark scenarios. Using plausible estimates for the achievable experimental efficiencies and the relevant background processes, we find that the prospective sensitivity of the diffractive Higgs production will allow to probe interesting regions of the $M_{A}-\tan \beta$ parameter plane of the MSSM. Central exclusive production of the $\mathcal{C P}$-even Higgs bosons of the MSSM may provide a unique opportunity to access the bottom Yukawa couplings of the Higgs bosons up to masses of $M_{H} \lesssim 250 \mathrm{GeV}$. We also discuss the prospects for identifying the $\mathcal{C} \mathcal{P}$-odd Higgs boson, $A$, in diffractive processes at the LHC.
\end{abstract}

*email: Sven.Heinemeyer@cern.ch

$\dagger$ email: V.A.Khoze@durham.ac.uk

$\ddagger$ email: Ryskin@MR11084.spb.edu

§email: W.J.Stirling@durham.ac.uk

^email: Marek.Tasevsky@cern.ch

" email: Georg.Weiglein@durham.ac.uk

**former address 


\section{Introduction}

Searches for Higgs bosons and the study of their properties are among the primary goals of the Large Hadron Collider (LHC) at CERN. For the Higgs boson of the Standard Model (SM) the discovery is, in principle, guaranteed for any mass [1-4]. Various extended models predict a large diversity of Higgs-like bosons with different masses, couplings and $\mathcal{C} \mathcal{P}$-parities. The most elaborate extension of the SM up to now is the Minimal Supersymmetric Standard Model (MSSM) [5], in which there are three neutral $(h, H$ and $A)$ and two charged $\left(H^{+}, H^{-}\right)$ Higgs bosons. At lowest order the Higgs sector of the MSSM is $\mathcal{C P}$-conserving, with the $\mathcal{C} \mathcal{P}$ even states $h$ and $H\left(M_{h}<M_{H}\right)$ and the $\mathcal{C P}$-odd state $A$. The Higgs sector of the MSSM is affected by large higher-order corrections (see for example Ref. [6] for recent reviews), which have to be taken into account for reliable phenomenological predictions.

Within the MSSM, the LHC will be able to observe all the Higgs states of the model over a significant part of the MSSM parameter space. There exists an important parameter region, however, where the LHC will detect only one of the MSSM Higgs bosons with SM-like properties. Revealing that a detected new state is indeed a Higgs boson and distinguishing the Higgs boson(s) of the SM or the MSSM from the states of extended Higgs theories will be non-trivial. This goal will require a comprehensive programme of precision Higgs measurements. In particular, it will be of utmost importance to determine the spin and $\mathcal{C P}$ properties of a new state and to measure precisely its mass, width and couplings.

While ultimately the cleaner experimental environment of electron-positron collisions will be required to assemble a comprehensive phenomenological profile of the Higgs sector [7-10], it will be highly important to fully exploit the experimental capabilities of the LHC. The "standard" LHC production channels are gluon fusion, weak boson fusion and associated production with heavy quarks or vector bosons. The accuracy in determining the mass of the new particle via these channels will depend on whether the $H \rightarrow \gamma \gamma$ or $H \rightarrow Z Z \rightarrow 4 \mu$ channels will be accessible. The observation of the new state in different channels will provide valuable information on its couplings [11-13] and will also enable initial studies of further properties [14].

There has been a great deal of attention devoted recently to the possibility of complementing the standard LHC physics menu by adding forward proton detectors to the CMS and ATLAS experiments (see for example Refs. [15-28] and references therein). The use of forward proton tagging would provide an exceptionally clean environment to search for new phenomena at the LHC and to identify their nature. Of particular interest in this context is "central exclusive diffractive" (CED) Higgs-boson production $p p \rightarrow p \oplus H \oplus p$, where the $\oplus$ signs are used to denote the presence of large rapidity gap 1 . In these exclusive processes there is no hadronic activity between the outgoing protons and the decay products of the central system. The predictions for exclusive production are obtained by calculating the diagram of Fig. 1 using techniques developed in Refs. [15, 16, 29].

There are several major reasons why central exclusive diffractive (CED) production is so attractive for Higgs boson studies. First, if the outgoing protons remain intact and scatter through small angles then, to a very good approximation, the primary active di-gluon system obeys a $J_{z}=0, \mathcal{C P}$-even selection rule $[30,31]$. Here $J_{z}$ is the projection of the

\footnotetext{
${ }^{1}$ We focus here on neutral Higgs-boson production. Charged Higgs bosons can also be exclusively produced in $p p$ collisions predominantly via the photon fusion mechanism $\gamma \gamma \rightarrow H^{+} H^{-}$.
} 


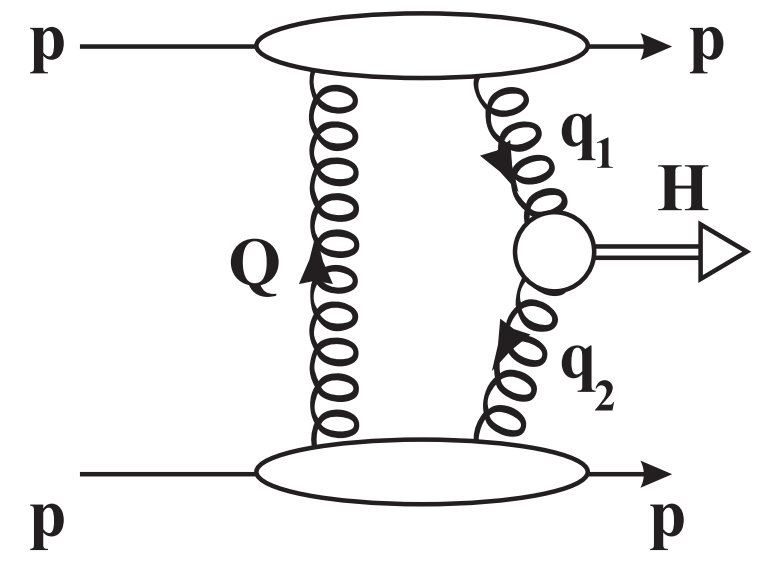

Figure 1: Schematic diagram for central exclusive diffractive (CED) Higgs production at the LHC, $p p \rightarrow p \oplus H \oplus p$.

total angular momentum along the proton beam axis. This selection rule readily permits a clean determination of the quantum numbers of the observed Higgs resonance which will be dominantly produced in a scalar state. Furthermore, because the process is exclusive, the energy loss of the outgoing protons is directly related to the mass of the central system, allowing a potentially excellent mass resolution, irrespective of the decay mode of the produced particle2. Another important feature of the CED process is that it may enable to a signal-to-background ratio of order 1 (or even better) to be achieved $[17,18]$. A particular advantage of forward proton tagging is that it would allow all the main Higgs-boson decay modes, $b \bar{b}, W W$ and $\tau \tau$, to be observed in this channel. It may in this way provide a unique possibility to study the Higgs coupling to bottom quarks, which may be difficult to access in other search channels at the LHC [4] despite the fact that $H \rightarrow b \bar{b}$ is by far the dominant decay mode for a light SM-like Higgs boson.

Within the MSSM, CED Higgs-boson production can be even more important than in the SM. The coupling of the lightest MSSM Higgs boson to bottom quarks and $\tau$ leptons can be strongly enhanced for large values of $\tan \beta$, the ratio of the vacuum expectation values of the two Higgs doublets in the MSSM Higgs sector, and relatively small values of the $\mathcal{C P}$-odd Higgs-boson mass, $M_{A}$. As a consequence, in this parameter region the expected Higgs signal-to-background ratios in the $b \bar{b}$ channel are much larger than for the corresponding SM process [33] (see also Ref. [34]). It is interesting to note in this context that in some MSSM scenarios CED production would provide the possibility for lineshape analyses (to discriminate between different Higgs-boson signals) [19,33] and offer a way for direct observation of a $\mathcal{C P}$-violating signal in the Higgs sector $[19,35]$. In a situation where the total width is larger than the mass resolution, the CED process may provide a unique opportunity to measure the total width.

The lightest MSSM Higgs boson becomes SM-like for larger values of $M_{A}$. For $M_{H} \approx$ $M_{A} \gtrsim 2 M_{W}$ the lightest MSSM Higgs boson couples to gauge bosons with about SM strength, while the heavy MSSM Higgs bosons, $H$ and $A$, decouple from the gauge bosons. The search

\footnotetext{
${ }^{2}$ Current studies suggest $[18,20,32]$ that the missing mass resolution will be of order $1 \%$ for Higgs masses above $120 \mathrm{GeV}$, assuming both protons are detected at $420 \mathrm{~m}$ from the interaction point.
} 
for heavy MSSM Higgs bosons therefore differs significantly from the case of a heavy SMlike Higgs boson. While for a SM-like Higgs boson the weak-boson fusion channel is a promising production process and the decay $H \rightarrow Z Z \rightarrow 4$ leptons is the "gold-plated" search channel $[1,4]$, none of these channels (nor Higgs boson decay into $W$ bosons) can be used in the search for heavy MSSM Higgs bosons. This leads to the result that in a significant part of the MSSM parameter space, the well-known "LHC wedge region" $[1,4,36]$, the heavy MSSM Higgs bosons escape detection at the LHC.

In CED the heavy $\mathcal{C P}$-even MSSM Higgs boson $H$ can be produced and its decay into $b \bar{b}$ can be utilised. While in the SM the $\mathrm{BR}(H \rightarrow b \bar{b})$ is strongly suppressed for $M_{H} \gtrsim 2 M_{W}$ because of the dominant decay into gauge bosons, in the MSSM $H \rightarrow b \bar{b}$ remains by far the dominant decay mode also for larger masses, as long as no decays into supersymmetric particles (or lighter Higgs bosons) are open. CED Higgs boson production with decay to $b \bar{b}$ is therefore important over a much larger mass range than in the SM. In this paper we study the prospects for the channels $h, H \rightarrow b \bar{b}$ and $h, H \rightarrow \tau^{+} \tau^{-}$in CED production and discuss the discovery reach of the various channels in the $M_{A}-\tan \beta$ parameter plane. We also analyse the channels $h, H \rightarrow W W^{(*)}$ and compare them with the SM case.

$\mathrm{CED}$ production of the $\mathcal{C P}$-odd Higgs boson is less promising than production of the $\mathcal{C P}$ even state because this mode is strongly suppressed by the $\mathcal{C P}$-even selection rule $[30,31]$. We therefore investigate the prospects of this channel in a less exclusive reaction and discuss the possibilities for distinguishing $A$ and $H$.

The outline of the paper is as follows. The Higgs sector of the MSSM is briefly described in Sect. 2, where we also define the benchmark scenarios used later for the numerical analysis. In Sect. 3 we review the relevant Higgs-boson production cross sections, while in Sect. 4 the various backgrounds are discussed. The experimental aspects of CED production of $\mathcal{C P}$-even MSSM Higgs bosons are discussed in Sect. 5. Our numerical analysis of the CED production processes of the $\mathcal{C} \mathcal{P}$-even MSSM Higgs bosons is presented in Sect. 6. In Sect. 7 we comment on the observability of the $\mathcal{C} \mathcal{P}$-odd MSSM Higgs boson. Our conclusions can be found in Sect. 8 ,

\section{The MSSM Higgs sector: notations and benchmark scenarios}

\section{$2.1 \quad$ Tree-level structure}

Unlike in the SM, in the MSSM two Higgs doublets are required. At the tree level, the Higgs sector can be described with the help of two independent parameters, usually chosen as the ratio of the two vacuum expectation values, $\tan \beta \equiv v_{2} / v_{1}$, and $M_{A}$, the mass of the $\mathcal{C} \mathcal{P}$-odd $A$ boson. Diagonalisation of the bilinear part of the Higgs potential, i.e. the Higgs mass matrices, is performed via rotations by angles $\alpha$ for the $\mathcal{C P}$-even part and $\beta$ for the $\mathcal{C} \mathcal{P}$-odd and the charged part. The angle $\alpha$ is thereby determined through

$$
\tan 2 \alpha=\tan 2 \beta \frac{M_{A}^{2}+M_{Z}^{2}}{M_{A}^{2}-M_{Z}^{2}} ; \quad-\frac{\pi}{2}<\alpha<0 .
$$


One obtains five physical states, the neutral $\mathcal{C} \mathcal{P}$-even Higgs bosons $h, H$, the $\mathcal{C} \mathcal{P}$-odd Higgs boson $A$, and the charged Higgs bosons $H^{ \pm}$. Furthermore there are three unphysical Goldstone boson states, $G^{0}, G^{ \pm}$. At lowest order, the Higgs-boson masses can be expressed in terms of $M_{Z}, M_{W}$, and $M_{A}$,

$$
\begin{aligned}
M_{h, H}^{2} & =\frac{1}{2}\left[M_{A}^{2}+M_{Z}^{2} \mp \sqrt{\left(M_{A}^{2}+M_{Z}^{2}\right)^{2}-4 M_{A}^{2} M_{Z}^{2} \cos ^{2} 2 \beta}\right], \\
M_{H^{ \pm}}^{2} & =M_{A}^{2}+M_{W}^{2} .
\end{aligned}
$$

In the decoupling limit, which is typically reached for $M_{A} \gtrsim 150 \mathrm{GeV}$ (depending on $\tan \beta$ ), the heavy MSSM Higgs bosons are nearly degenerate in mass, $M_{A} \approx M_{H} \approx M_{H^{ \pm}}$. The couplings of the neutral Higgs bosons to SM gauge bosons are proportional to

$$
V V h \sim \sin (\beta-\alpha), V V H \sim \cos (\beta-\alpha), \quad\left(V=Z, W^{ \pm}\right)
$$

while the coupling $V V A \equiv 0$ at tree level. In the decoupling limit one finds $\beta-\alpha \rightarrow \pi / 2$, i.e. $\sin (\beta-\alpha) \rightarrow 1, \cos (\beta-\alpha) \rightarrow 0$. Consequently, for $M_{A} \gtrsim 150 \mathrm{GeV}$ one finds the following decay patterns for the neutral MSSM Higgs bosons at tree-level:

$h$ : the light $\mathcal{C P}$-even Higgs boson has SM-like decays. Due to its upper mass limit of $M_{h} \lesssim 130 \mathrm{GeV}[37,38]$ (see below), a non-negligible decay to gauge bosons only occurs in a limited window of $M_{h}$ values close to this upper limit.

$H, A$ : compared to a SM Higgs boson with mass $M_{H^{\mathrm{SM}}} \gtrsim 150 \mathrm{GeV}$, which would decay predominantly into SM gauge bosons, the decays of $H, A$ to SM gauge bosons are strongly suppressed. In turn, the branching ratios to $b \bar{b}$ and $\tau^{+} \tau^{-}$are much larger in this mass range compared to the SM case. As a rule of thumb, $\mathrm{BR}(H, A \rightarrow b \bar{b}) \approx 90 \%$ and $\operatorname{BR}\left(H, A \rightarrow \tau^{+} \tau^{-}\right) \approx 10 \%$, if SUSY particles (such as charginos and neutralinos) are too heavy to be produced in the decays of $H$ and $A$.

\subsection{Higher-order corrections}

Higher-order corrections in the MSSM Higgs sector are in general quite large. In particular, higher-order corrections give rise to an upward shift of the upper bound on the light $\mathcal{C P}$-even Higgs-boson mass from the tree-level value, $M_{h} \leq M_{Z}$, to about $M_{h} \lesssim 130 \mathrm{GeV}[38,39]$. Besides the impact on the masses, large higher-order corrections also affect the Higgs-boson couplings. For the evaluation of the theoretical predictions for the relevant observables in the MSSM Higgs sector we use the code FeynHiggs [37-40].

For simplicity, in the following we confine our attention to the $\mathcal{C P}$-conserving case, i.e. we do not consider $\mathcal{C} \mathcal{P}$-violating complex phases. However the analysis in this paper can easily be extended to the case of non-vanishing complex phases. In order to fix our notation, we list the conventions for the inputs from the scalar top and scalar bottom sector of the MSSM: the mass matrices in the basis of the current eigenstates $\tilde{t}_{L}, \tilde{t}_{R}$ and $\tilde{b}_{L}, \tilde{b}_{R}$ are given by

$$
\mathcal{M}_{\tilde{t}}^{2}=\left(\begin{array}{cc}
M_{\tilde{Q}}^{2}+m_{t}^{2}+\cos 2 \beta\left(\frac{1}{2}-\frac{2}{3} s_{\mathrm{w}}^{2}\right) M_{Z}^{2} & m_{t} X_{t} \\
m_{t} X_{t} & M_{\tilde{t}_{R}}^{2}+m_{t}^{2}+\frac{2}{3} \cos 2 \beta s_{\mathrm{w}}^{2} M_{Z}^{2}
\end{array}\right),
$$




$$
\mathcal{M}_{\tilde{b}}^{2}=\left(\begin{array}{cc}
M_{\tilde{Q}}^{2}+m_{b}^{2}+\cos 2 \beta\left(-\frac{1}{2}+\frac{1}{3} s_{\mathrm{w}}^{2}\right) M_{Z}^{2} & m_{b} X_{b} \\
m_{b} X_{b} & M_{\tilde{b}_{R}}^{2}+m_{b}^{2}-\frac{1}{3} \cos 2 \beta s_{\mathrm{w}}^{2} M_{Z}^{2}
\end{array}\right),
$$

where

$$
m_{t} X_{t}=m_{t}\left(A_{t}-\mu \cot \beta\right), \quad m_{b} X_{b}=m_{b}\left(A_{b}-\mu \tan \beta\right) .
$$

Here $A_{t}$ denotes the trilinear Higgs-stop coupling, $A_{b}$ denotes the Higgs-sbottom coupling, and $\mu$ is the higgsino mass parameter. As an abbreviation we will use

$$
M_{\mathrm{SUSY}} \equiv M_{\tilde{Q}}=M_{\tilde{t}_{R}}=M_{\tilde{b}_{R}} .
$$

The relation between the bottom-quark mass and the Yukawa coupling $h_{b}$, which also controls the interaction between the Higgs fields and the sbottom quarks, reads at lowest order $m_{b}=h_{b} v_{1}$. This relation is affected at one-loop order by large radiative corrections [41-44], proportional to $h_{b} v_{2}$, in general giving rise to $\tan \beta$-enhanced contributions. These terms proportional to $v_{2}$ are generated either by gluino-sbottom one-loop diagrams (resulting in $\mathcal{O}\left(\alpha_{b} \alpha_{s}\right)$ corrections, $\alpha_{b} \equiv h_{b}^{2} /(4 \pi)$ ), or by chargino-stop loops (giving $\mathcal{O}\left(\alpha_{b} \alpha_{t}\right)$ corrections, $\alpha_{t} \equiv h_{t}^{2} /(4 \pi)$ with $h_{t}$ being the top Yukawa coupling). Because the $\tan \beta$-enhanced contributions can be numerically relevant, an accurate prediction of $h_{b}$ from the experimental value of the bottom mass requires a resummation of such effects to all orders in the perturbative expansion, as described in Refs. [42,43].

The leading effects are included in the effective Lagrangian formalism developed in Ref. [42]. Numerically this is by far the dominant part of the contributions from the sbottom sector (see also Refs. [45-47]). The effective Lagrangian is given by

$$
\begin{aligned}
& \mathcal{L}=\frac{g}{2 M_{W}} \frac{\bar{m}_{b}}{1+\Delta_{b}}\left[\quad \tan \beta A i \bar{b} \gamma_{5} b+\sqrt{2} V_{t b} \tan \beta H^{+} \bar{t}_{L} b_{R}\right. \\
& +\left(\frac{\sin \alpha}{\cos \beta}-\Delta_{b} \frac{\cos \alpha}{\sin \beta}\right) h \bar{b}_{L} b_{R} \\
& \left.-\left(\frac{\cos \alpha}{\cos \beta}+\Delta_{b} \frac{\sin \alpha}{\sin \beta}\right) H \bar{b}_{L} b_{R}\right]+ \text { h.c. . }
\end{aligned}
$$

Here $\bar{m}_{b}$ denotes the running bottom quark mass including SM QCD corrections. The prefactor $1 /\left(1+\Delta_{b}\right)$ in eq. (8) arises from the resummation of the leading corrections to all orders. The additional terms $\sim \Delta_{b}$ in the $h \bar{b} b$ and $H \bar{b} b$ couplings arise from the mixing and coupling of the "other" Higgs boson, $H$ and $h$ respectively, to the $b$ quarks.

As explained above, the function $\Delta_{b}$ consists of two main contributions, an $\mathcal{O}\left(\alpha_{s}\right)$ correction from a sbottom-gluino loop and an $\mathcal{O}\left(\alpha_{t}\right)$ correction from a stop-higgsino loop. The explicit form of $\Delta_{b}$ in the limit of $M_{\mathrm{SUSY}} \gg m_{t}$ and $\tan \beta \gg 1$ reads [41]

$$
\Delta_{b}=\frac{2 \alpha_{s}}{3 \pi} m_{\tilde{g}} \mu \tan \beta \times I\left(m_{\tilde{b}_{1}}, m_{\tilde{b}_{2}}, m_{\tilde{g}}\right)+\frac{\alpha_{t}}{4 \pi} A_{t} \mu \tan \beta \times I\left(m_{\tilde{t}_{1}}, m_{\tilde{t}_{2}}, \mu\right) .
$$

The function $I$ is given by

$$
I(a, b, c)=\frac{1}{\left(a^{2}-b^{2}\right)\left(b^{2}-c^{2}\right)\left(a^{2}-c^{2}\right)}\left(a^{2} b^{2} \log \frac{a^{2}}{b^{2}}+b^{2} c^{2} \log \frac{b^{2}}{c^{2}}+c^{2} a^{2} \log \frac{c^{2}}{a^{2}}\right)
$$




$$
\sim \frac{1}{\max \left(a^{2}, b^{2}, c^{2}\right)} .
$$

The sign of $\Delta_{b}$ is governed by the sign of the parameter $\mu$ (and for the second term of eq. (9) also by the sign of $A_{t}$ ). As a consequence of eq. (8), positive values of $\Delta_{b}$ lead to a suppression of the bottom Yukawa coupling. On the other hand, for negative values of $\Delta_{b}$ the bottom Yukawa coupling may be strongly enhanced (and can even acquire non-perturbative values when $\left.\Delta_{b} \rightarrow-1\right)$. The CED channel, $p p \rightarrow p \oplus H \oplus p$ with $H \rightarrow b \bar{b}$, receives important contributions from the $\Delta_{b}$ corrections via the bottom Yukawa coupling. We will in the following discuss the impact of the $\Delta_{b}$ corrections by considering different values of the parameter $\mu$.

Another important source of higher-order corrections are Higgs-propagator corrections. They affect the Higgs-boson masses (as discussed above) and all Higgs-boson couplings. In the coupling of the light $\mathcal{C P}$-even Higgs boson to bottom quarks, for example, the Higgspropagator corrections lead, to a good approximation, to the replacement [48]

$$
h b \bar{b} \sim y_{b} \frac{\sin \alpha}{\cos \beta} \rightarrow y_{b} \frac{\sin \alpha_{\text {eff }}}{\cos \beta},
$$

where $\alpha_{\text {eff }}$ contains the contributions from the Higgs-boson propagator corrections [48]. For certain parts of the MSSM parameter space, up to $M_{A} \lesssim 350 \mathrm{GeV}$, it is possible that $\alpha_{\text {eff }} \rightarrow 0$, and thus the coupling of the light Higgs boson to bottom quarks becomes tiny [48]. An example of a scenario where this is realised is the "small $\alpha_{\text {eff }}$ " scenario, as discussed in the next subsection.

\subsection{Benchmark scenarios - bounds from Higgs-boson searches}

Due to the large number of MSSM parameters, a number of benchmark scenarios $[49,50]$ have been used for the interpretation of MSSM Higgs boson searches at LEP $[51,52]$ and at the Tevatron [53-57].

Since at tree level the Higgs sector of the MSSM is governed by two parameters (in addition to $M_{Z}$ and the SM gauge couplings), the definition of the benchmarks is such that the two tree-level parameters, $M_{A}$ and $\tan \beta$, are varied while the values of all other parameters are fixed at certain benchmark settings. From the most commonly used benchmark scenarios for the $\mathcal{C P}$-conserving MSSM from Refs. $[49,50]$ we list here the three scenarios that are relevant for our analysis.

- The $M_{h}^{\max }$ scenario:

In this scenario the parameters are chosen such that the mass of the light $\mathcal{C} \mathcal{P}$-even Higgs boson acquires its maximum possible values as a function of $\tan \beta$ (for fixed $M_{\mathrm{SUSY}}$, $m_{t}$ and $M_{A}$ set to its maximum value, $M_{A}=1 \mathrm{TeV}$ ). This was used in particular to obtain conservative $\tan \beta$ exclusion bounds [58] at LEP [52]. The parameters are 3 :

$$
\begin{aligned}
& m_{t}=172.7 \mathrm{GeV}, \quad M_{\mathrm{SUSY}}=1 \mathrm{TeV}, \quad \mu=200 \mathrm{GeV}, \quad M_{2}=200 \mathrm{GeV}, \\
& X_{t}=2 M_{\mathrm{SUSY}} \quad A_{b}=A_{t}, \quad m_{\tilde{g}}=0.8 M_{\mathrm{SUSY}} .
\end{aligned}
$$

\footnotetext{
${ }^{3}$ Using instead the current experimental central top quark mass value of $m_{t}=170.9 \mathrm{GeV}$ [59] would have only a minor impact on our analysis.
} 
- The no-mixing scenario:

This benchmark scenario is the same as the $M_{h}^{\max }$ scenario, but with vanishing mixing in the $\tilde{t}$ sector and with a higher SUSY mass scale (the latter has been chosen to avoid conflict with the exclusion bounds from the LEP Higgs searches $[51,52]$ ),

$$
\begin{aligned}
& m_{t}=172.7 \mathrm{GeV}, \quad M_{\mathrm{SUSY}}=2 \mathrm{TeV}, \quad \mu=200 \mathrm{GeV}, \quad M_{2}=200 \mathrm{GeV}, \\
& X_{t}=0 \quad A_{b}=A_{t}, \quad m_{\tilde{g}}=0.8 M_{\mathrm{SUSY}} .
\end{aligned}
$$

- The small- $\alpha_{\text {eff }}$ scenario:

As explained above, the decays $h \rightarrow b \bar{b}$ (and also $h \rightarrow \tau^{+} \tau^{-}$) can be strongly affected by corrections entering via the effective mixing angle $\alpha_{\text {eff }}$. If $\alpha_{\text {eff }}$ is small, these two decay channels can be strongly suppressed in the MSSM due to the additional factor $-\sin \alpha_{\text {eff }} / \cos \beta$ compared to the SM coupling. Such a suppression occurs for large $\tan \beta$ and not too large $M_{A}$ for the following parameters:

$$
\begin{aligned}
& m_{t}=172.7 \mathrm{GeV}, \quad M_{\mathrm{SUSY}}=800 \mathrm{GeV}, \quad \mu=2.5 M_{\mathrm{SUSY}}, \quad M_{2}=500 \mathrm{GeV} \\
& X_{t}=-1100 \mathrm{GeV}, \quad A_{b}=A_{t}, \quad m_{\tilde{g}}=500 \mathrm{GeV} .
\end{aligned}
$$

As discussed above, in order to study the impact of potentially large corrections in the $b / \tilde{b}$ sector it is useful to vary the absolute value and sign of the parameter $\mu$. This has led to the definition of an extension of the $M_{h}^{\max }$ and the no-mixing scenario by the following values of $\mu[50]$

$$
\mu= \pm 200, \pm 500, \pm 1000 \mathrm{GeV},
$$

allowing both an enhancement and a suppression of the bottom Yukawa coupling and taking into account the limits from direct searches for charginos at LEP.

The other MSSM parameters that are not specified above have only a minor impact on MSSM Higgs-boson phenomenology. In our numerical analysis below we fix them such that all soft SUSY-breaking parameters in the diagonal entries of the sfermion mass matrices are set to $M_{\mathrm{SUSY}}$, and the trilinear couplings for all sfermions are set to $A_{t}$.

For the exclusion bounds from the LEP Higgs searches the channel $e^{+} e^{-} \rightarrow Z^{*} \rightarrow Z h, H$ played a major role. We will indicate the bounds obtained from this channel (for fixed $M_{\mathrm{SUSY}}$ and $\left.m_{t}\right)$ [52] in our figures below. As expected, the bounds are weaker in the $M_{h}^{\max }$ scenario compared to the no-mixing scenario. Limits from Run II of the Tevatron have been published for the following channels [53-57] $(\phi=h, H, A)$ :

$$
\begin{aligned}
& p \bar{p} \rightarrow b \bar{b} \phi, \phi \rightarrow b \bar{b} \text { (with one additional tagged } b \text { jet) } \\
& p \bar{p} \rightarrow \phi \rightarrow \tau^{+} \tau^{-} \text {(inclusive), } \\
& p \bar{p} \rightarrow t \bar{t} \rightarrow H^{ \pm} W^{\mp} b \bar{b}, H^{ \pm} \rightarrow \tau \nu_{\tau} .
\end{aligned}
$$

While these limits begin to probe the region of small $M_{A}$ and large $\tan \beta$ that is of particular interest for the CED Higgs production analyses performed in this paper, the parameter region with $M_{A} \gtrsim 100 \mathrm{GeV}$ and $\tan \beta \lesssim 50$ currently remains unaffected by the Tevatron exclusion bounds. 


\section{Cross sections for CED Higgs production in the MSSM}

In what follows we use the formalism of Refs. $[15,16,31]$ to obtain the cross sections for CED production of Higgs bosons, similarly to Ref. [33]. The amplitudes, corresponding to the diagram of Fig. 1, are calculated using perturbative QCD techniques $[15,16]$. There is also the possibility of soft rescattering in which particles from the underlying events populate the gaps. Accounting for these absorptive effects leads to a rather small probability for the survival of the rapidity gaps, the so-called "survival factor" $S^{2}[60,61]$. A typical value for $S^{2}$ is about $S^{2}=0.025$, determined from summation of multi-pomeron amplitudes [60].

For the purposes of this paper it is sufficient to use simple approximate formulae for the Higgs signal and background cross sections as a function of mass, derived in Refs. [33,62]. At LHC energies these formulae approximate the full results of Ref. [33] with an accuracy of better than $10 \%$ for Higgs masses in the region $50 \mathrm{GeV} \lesssim M_{h}, M_{H} \lesssim 350 \mathrm{GeV}$. For a more detailed analysis the formalism of Refs. [15,16,31] should be applied and appropriate MC programs, such as ExHuME (see Refs. [63,64]), together with ATLAS or CMS simulation programs should be used to account for detector effects.

Here and in what follows we evaluate the cross sections $\sigma^{\text {excl }}$ for CED production of $h, H$ using the simplified formula

$$
\sigma^{\text {excl }} \mathrm{BR}^{\mathrm{MSSM}}=3 \mathrm{fb}\left(\frac{136}{16+M}\right)^{3.3}\left(\frac{120}{M}\right)^{3} \frac{\Gamma(h / H \rightarrow g g)}{0.25 \mathrm{MeV}} \mathrm{BR}^{\mathrm{MSSM}},
$$

where the gluonic partial width $\Gamma(h / H \rightarrow g g)$ and the branching ratios for the various channels in the MSSM, BR ${ }^{\mathrm{MSSM}}$, are calculated using the program FeynHiggs [37-40]. The mass $M$ (in $\mathrm{GeV}$ ) represents the Higgs-boson mass, i.e. $M=M_{h}$ for $h$ and $M=M_{H}$ for $H$. The factor $(136 /(16+M))^{3.3}$ accounts for the mass dependence of the effective "exclusive" $g g^{P P}$ luminosity, see Refs. [33,62]. The normalization is fixed at $M=120 \mathrm{GeV}$, where in accordance with Ref. [33] we obtain $\sigma^{\text {excl }}=3 \mathrm{fb}$ with the width $\Gamma\left(H^{\mathrm{SM}} \rightarrow g g\right)=0.25 \mathrm{MeV}$. In Ref. [33] various uncertainties in the prediction of the CED cross sections were evaluated. Combining different sources of possible uncertainties in the gluon-gluon $g g^{P P}$ luminosity, it was found that the normalisation of $3 \mathrm{fb}$ may be uncertain to a factor of about 2.5. One particular source of uncertainty is the sensitivity to the gluon distribution, which on its own generates a factor of 1.5 uncertainty. We anticipate that an accurate calibration of the $g g^{P P}$ luminosity at the LHC will be obtained by measuring exclusive high $E_{T}$ dijet (or exclusive $\gamma \gamma$ ) production, see Refs. [31,65]. Note also that the existing CDF data on measurements of such events at the Tevatron [66] are in reasonable quantitative agreement with the expectations [21] using exactly the same formalism $[16,31]$ that we are using to obtain the cross sections for CED Higgs-boson production 4 .

Eq. (19) yields a total number of signal events of about 100 for a SM Higgs boson with $M_{H^{\mathrm{Sm}}}=120 \mathrm{GeV}$ with an integrated luminosity of $60 \mathrm{fb}^{-1}$ if only the forward detector acceptances are accounted for and no cuts and efficiences in the central detector are imposed.

In the case of pseudoscalar $A$ production, which is suppressed by the $\mathcal{C P}$-even selection

\footnotetext{
${ }^{4}$ Additional uncertainties in the production cross sections of up to $\sim 20 \%$ could arise for large tan $\beta$ due to the imperfect inclusion of NNLO QCD corrections.
} 
rule (see Refs. $[30,31]$ ), the signal cross section is given approximately by

$$
\sigma^{\mathrm{excl}} \mathrm{BR}^{\mathrm{MSSM}}=3 \mathrm{fb}\left(0.0071 \frac{136^{3.3}}{(0.5+M)^{3.45}}\right)\left(\frac{120}{M}\right)^{3} \frac{\Gamma(A \rightarrow g g)}{0.25 \mathrm{MeV}} \mathrm{BR}^{\mathrm{MSSM}}
$$

with $M=M_{A}$. Again, the gluonic partial width $\Gamma(A \rightarrow g g)$ and $A$ decay branching ratios are evaluated with the program FeynHiggs [37-40].

\section{Background processes}

\subsection{Summary of the backgrounds to the $p \oplus(h, H \rightarrow b \bar{b}) \oplus p$ signal}

As we have already discussed, the advantage of the $p \oplus(h, H \rightarrow b \bar{b}) \oplus p$ signal is that there exists a $J_{z}=0$ selection rule, which requires the leading order $g g^{P P} \rightarrow b \bar{b}$ QCD background subprocess to vanish in the limit of massless quarks and forward outgoing protons. However, there are still various sources of potentially important background contributions, for more details see Refs. [17,21,67].

As discussed in Ref. [67] it is convenient to consider separately the quark helicity conserving (QHC) and the quark helicity non-conserving (QHNC) background amplitudes. These amplitudes do not interfere, and their contributions can be treated independently which, in particular, avoids double-counting.

There are four main types of background subprocess contributions.

(i) The prolific $(\mathrm{LO}) g g^{P P} \rightarrow g g$ subprocess can mimic $b \bar{b}$ production since one may misidentify the outgoing gluons as $b$ and $\bar{b}$ jets. This contribution was first evaluated in Ref. [17] assuming a $1 \%$ probability of misidentification per jet and applying a $60^{\circ}<\theta<120^{\circ}$ jet cut.

(ii) An admixture of $\left|J_{z}\right|=2$ production, arising from non-forward going protons, which contributes to the $(\mathrm{QHC}) \mathrm{LO} g g^{P P} \rightarrow b \bar{b}$ background.

(iii) Since the $b$-quarks have non-zero mass there is a contribution to the $J_{z}=0$ (QHNC) cross section of order $m_{b}^{2} / E_{T}^{2}$. It is this term which currently raises the main concern from the theory side. The problem is that the result is strongly affected by the (uncomfortably large) higher-order QCD effects which can dominate over the Born-level prediction, see Refs. [67,68]. In particular, the one-loop double logarithmic contribution is larger than the Born term, and the final result becomes strongly dependent on the NNLO effects as well as on the scale $\mu$ at which the QCD coupling $\alpha_{S}$ is evaluated, and on the running $b$ quark mass. Although no complete result for these higher-order effects to the $g g^{P P} \rightarrow b \bar{b}$ process currently exists, an estimate has been given in Ref. [67] based on a seemingly plausible hypothesis that the NNLO effects can be incorporated in a way similar to the simpler process $\gamma \gamma \rightarrow q \bar{q} g$, where the full one-loop result is known. However without the complete result for the higher-order radiative corrections corresponding to the $g g^{P P} \rightarrow b \bar{b}$ amplitude it is impossible to make a firm prediction.

It is our understanding that the estimate of this contribution, given in Ref. [67], should be valid to an accuracy not better than a factor of 2-4 (or maybe even worse), and this 
exclusive piece currently represents the main source of theoretical uncertainty in the predictions for the overall background coming from single proton-proton collisions. A welcome feature of this contribution is that it decreases with increasing $E_{T}$ much faster than the other background terms, see below and Refs. [33,35].

(iv) Finally, there is the possibility of NLO $g g^{P P} \rightarrow b \bar{b} g$ (dominantly QHC) background contributions, which for hard gluon radiation at large angles do not obey the selection rules, see Refs. $[17,67,69]$. Of course the extra gluon can in principle be observed experimentally (as an additional jet) and the contribution of such background events reduced. However, there are important exceptions which are discussed in detail in Refs. [17,67].

(a) The extra gluon may go unobserved in the direction of a forward proton. This background may be reduced by requiring the approximate equality $M_{\text {missing }}=$ $M_{b \bar{b}}$. The degree of this reduction depends on the mass resolution in the proton detector and jet energy resolution in the central detector. The calculations in Ref. [70] show that for the $b \bar{b}$ mass interval $\Delta M_{b b}=0.2 M_{b \bar{b}}$ and a mass window of $\Delta M=5 \mathrm{GeV}$ ( $\Delta M$ indicates the mass window over which the signal and background are collected) the background arising from these forward going gluons (with $k_{T}<5 \mathrm{GeV}$ ) does not exceed $5 \%$ of the SM Higgs signal and, therefore, can be safely neglected.

(b) The remaining danger is large-angle hard gluon emission which is collinear with either the $b$ or $\bar{b}$ jet, and, therefore, unobservable. A general study of a background coming from three-jet $b \bar{b} g$ production has been performed in Ref. [67]. This background source results in a sizable contribution and is therefore taken into account in the final background formula, see eq. (21) below.

In principle, we also have to consider the higher-order contributions corresponding to the radiation of additional (soft) gluons. These terms have to be taken into account together with the virtual loop radiative corrections to the lowest-order cross section discussed in Refs. [16,67]. However, numerically, these contributions do not signficantly increase the radiative background, see Ref. [70]. It should be noted that the effect of gluon emission off the screening (labeled "Q" in Fig. 11) is also numerically small [71].

There are also some other potentially worrying background sources, which after a thorough investigation $[67,70]$ have not been included in the final expression for the $b \bar{b}$ background in eq. (21) below. This is either because their contributions are numerically small from the very beginning, or because they can be reduced to an acceptable level by straightforward experimental cuts. First, there is the NNLO QHC contribution to the exclusive process which comes from the one-loop box diagrams. This piece is not mass-suppressed and is potentially important especially for large $M_{H}$. As a consequence of rotational invariance (see Ref. [69]) it has a different angular dependence than the other contributions. In particular, this term vanishes at $\theta=\pi / 2$ and reaches its maximum at $\theta=\pi / 4$. However, numerically, this contribution is comparatively small.

Second, a potential background source can arise from the interaction of two soft Pomerons. This can result in the two main event categories: 
(a) central Higgs-boson production accompanied by two (or more) additional gluon jets,

(b) production of a high $E_{T} b \bar{b}$-pair accompanied by gluon jets.

In these cases the Higgs boson or the $b \bar{b}$ pair are produced in the collision of two gluons (from the Pomeron wave functions) via the hard subprocesses $(g g \rightarrow H$ or $g g \rightarrow b \bar{b})$ similarly to the usual inelastic event. In both processes the mass, $M_{b b}$, of the central $b \bar{b}$ system (resulting either from the Higgs decay or from the QCD background) is not equal to the 'PomeronPomeron' mass $M_{P P}=M_{\text {missing }}$, which is measured with good accuracy by the forward proton detectors. As discussed in Ref. [17], the suppression of such backgrounds is controlled by the requirement that $\left|M_{\text {missing }}-M_{b b}\right|$ should lie within the $\Delta M_{b b}$ mass interval. Unfortunately, the currently expected accuracy of the mass measurement in the central detector is not as good as anticipated in Ref. [17], and more detailed studies are therefore needed in order to evaluate these backgrounds more carefully, see Ref. [70]. To retain sufficient Higgs signal statistics we take the mass interval to be twice the mass resolution in the central detector, $\Delta M_{b b} \simeq 24 \mathrm{GeV}$. Already this requirement imposes a strong restriction on the diffractive parton distribution function (DPDF): the longitudinal momentum fraction $\beta$ of the Pomeron should be comparatively large, $\beta>0.6-0.7$. At the same time, at the large scales $(\mu \sim$ $\left.M_{H} / 2\right)$ relevant for Higgs production, the diffractive gluon distribution vanishes rapidly for $\beta \rightarrow 1$. As a result, the expected background cross sections appear to be quite small. Using the MRW2006 DPDFs [72] and imposing a cut $60^{\circ}<\theta<120^{\circ}$ on the final-state jets, we arrive at the following conclusion: for $\Delta M_{b b} \simeq 24 \mathrm{GeV}$ the $g b \bar{b} g$ and $g H g$ contributions are each less than about $6 \%$ of the SM Higgs signa 5 . Moreover, a sizable fraction of such events can be further rejected by observing the extra (gluon) jets in the detectors.

It should be noted that one could try to generate events produced in the collision of two soft Pomerons using the POMWIG Monte Carlo [73] (modulo a proper account of the soft survival factor $S^{2}$ ), see for instance Ref. [26]. Unfortunately, the published version of this generator uses the old H1 DPDFs (at the scale $\mu^{2} \sim 75 \mathrm{GeV}^{2}$ ), which are almost flat for $\beta>0.5$. At present, the large $\beta$ behaviour of the DPDFs is not sufficiently well constrained by the inclusive diffractive DIS data. In the new H1 QCD analysis [74] a good description of the diffractive DIS data is obtained by two fits: H1 2006 DPDF Fit A with essentially flat gluons and H1 2006 DPDF Fit B, where the diffractive gluon density rapidly decreases for $\beta>0.5$. However, it is only Fit $\mathrm{B}$ that is consistent with the HERA data on diffractive charm and dijet production. At the same time, this fit is quite close to the MRW2006 [72] results, described in terms of the LO perturbative QCD calculations. This provides a justification for our choice of the large $\beta$ behaviour of the DPDFs, and we therefore conclude that the background arising from soft Pomeron-Pomeron interactions can safely be neglected.

Finally, note that the exclusive Higgs signal may be smeared by the additional contribution coming from the CED $H+n \cdot g$ production process. However this piece is suppressed by colour constraints, since the $t$-channel two-gluon exchange across the gap region should be colourless. In particular, because of this there is no single gluon radiation. The first nonzero contribution starts from $n=2$, but this is additionally colour-suppressed since the pair

\footnotetext{
${ }^{5}$ It should be noted that since the diffractive gluon density $g^{D}\left(\beta, x_{\mathrm{Pom}}, \mu\right)$ (in our case $x_{\mathrm{Pom}}=\xi$ ) vanishes at least as $g^{D}\left(\beta, x_{\text {Pom }}, \mu\right) \propto(1-\beta)$ for $\beta \rightarrow 1$, the $g H g$ and $g b \bar{b} g$ contributions fall with decreasing $\Delta M_{b b}$ as $\left(\Delta M_{b b} / M_{b b}\right)^{k}$ with $k>4$. Two powers come from the phase space limitation, and two powers come from the behaviour of the diffractive gluons $\left(g^{D}\right)$ at large $\beta$.
} 
of gluons should form a colour singlet. Next, the mass of the $g H g$ system measured by forward protons is larger than that of the Higgs boson, and we have to impose the mass matching condition discussed in item (iv) above. Furthermore, those gluons with not too small transverse momenta, e.g. $k_{T}>5 \mathrm{GeV}$, can be detected and therefore such events can be rejected. Numerically, this background appears to be small (about 15\% of the SM Higgs signal [70]) and, again, it is not included in the final formula eq. (21) below. Therefore, the remaining significant background contributions come from exclusive dijet production, listed in items (i-iii, iv(b)) above 6 .

Within the accuracy of the existing calculations $[17,31,67]$, the overall background to the $0^{+}$Higgs signal in the $b \bar{b}$ mode can be approximated by the following formula

$$
\frac{\mathrm{d} \sigma^{B}}{\mathrm{~d} M} \approx 0.5 \mathrm{fb} / \mathrm{GeV}\left[0.92\left(\frac{120}{M}\right)^{6}+\frac{1}{2}\left(\frac{120}{M}\right)^{8}\right]
$$

where the first term in the square brackets corresponds to the processes listed in items (i-ii, iv (b)) above, while the last term arises from the mass-suppressed term described in item (iii).

It is worth mentioning that in the derivation of eq. (21) a $b$-jet cut $\left(60^{\circ}<\theta<120^{\circ}\right.$ in the $b \bar{b}$ rest frame) was applied to suppress the collinear singularities. In order to use the same efficiencies listed in Tab. 1 below for both the signal and background, we fix the normalization in eq. (21) as if the background had the same (flat) distribution in $\cos \theta$ as the Higgs decay signa 7 . Correspondingly, the value of $\sigma^{B}$ is normalised such that the contribution coming from the $60^{\circ}<\theta<120^{\circ}$ interval becomes equal to the genuine QCD cross section integrated over this $\theta$ interval.

It should be noted that in comparison with the background studies in Ref. [17], in eq. (21) we use a higher value of the probability $P_{g / b}$ for misidentifying a gluon as a $b$-jet, $P_{g / b}=1.3 \%$, corresponding to the values used in ATLAS studies $[1]$.

Recall that currently the most serious background is caused by exclusive $b \bar{b}$ QCD production, which has exactly the same characteristics as the $h / H \rightarrow b \bar{b}$ signal. The last term in eq. (21) shows the lowest-order result. As discussed above, the higher-order $\alpha_{s}$ corrections can be quite large. At the moment, only double-logarithmic effects are known, see Ref. [67] and references therein. It would therefore be desirable to calculate, at least, the complete one-loop expression for the $g g^{P P} \rightarrow b \bar{b}$ background.

Finally it is worth noticing that we use eq. (21) only for the numerical evaluations of the background contribution, and no detector simulation has been performed. In a more complete treatment, involving a detector simulation, optimisation procedures could be applied which would potentially further reduce the effects of backgrounds.

\footnotetext{
${ }^{6}$ We assume that multi-jet events can be rejected with the help of the central detector or one of the forward detectors.

7 This approximation proves to be sufficient within the given limited angular interval for $\theta\left(60^{\circ}<\theta<\right.$ $\left.120^{\circ}\right)$.

8 One may expect that in the particularly clean environment of the CED events, the value of $P_{g / b}$ could be reduced.
} 


\subsection{The backgrounds to the $p \oplus\left(h, H \rightarrow \tau^{+} \tau^{-}\right) \oplus p$ signal}

Although the $\tau^{+} \tau^{-}$signal has the advantage that there is practically no irreducible QCD background, there are still other sources of background events, see for example Ref. [35]. First, exclusive $\tau^{+} \tau^{-}$events may be produced by $\gamma \gamma$ fusion. Secondly, there may be a contribution caused by exclusive production of a pair of high $E_{T}(\sim M / 3)$ gluons being misidentified as a $\tau^{+} \tau^{-}$-pair. In more detail:

(a) The cross section for the QED production mechanism is appreciable, and is enhanced by two large logarithms arising from the integration over the transverse momenta of the exchanged photons. The corresponding luminosity factor can be calculated with sufficient accuracy using the equivalent photon approximation, see for example Refs. [16,75]. To suppress the contribution caused by a collinear singularity in the $\gamma \gamma \rightarrow \tau^{+} \tau^{-}$cross section, we impose a cut $60^{\circ}<\theta<120^{\circ}$ on the polar angle $\theta$ in the $\tau^{+} \tau^{-}$rest frame. As in the $b \bar{b}$ case, this results in a reduction of the Higgs signal by a factor of 2 . Neglecting the $\tau$-lepton mass and following the standard calculational procedure we obtain for the LHC energy $\sqrt{s}=14 \mathrm{TeV}$

$$
\sigma\left(p p \rightarrow p \oplus \tau^{+} \tau^{-} \oplus p\right)=20 \mathrm{fb} \frac{2 \Delta M}{M}\left(\frac{120 \mathrm{GeV}}{M}\right)^{2} .
$$

In eq. (22) we account for the gap survival factor $S^{2} \simeq 0.9$, caused by the 'soft' rescatterings (see Refs. $[60,75]$ ), and for reference purposes we use the cross section for exclusive production of a $\tau^{+} \tau^{-}$-pair with the invariant mass $M=120 \mathrm{GeV}$. For the mass window $\Delta M=5 \mathrm{GeV}$ at $M=120 \mathrm{GeV}$ the expected QED background is about $2 \mathrm{fb}$. Since the dominant contribution to the photon-photon luminosity comes from very small transverse momenta of the exchanged photons, to suppress this background we can select events with comparatively large $p_{T}$ of the outgoing protons. For example, if $p_{T}>200 \mathrm{MeV}$, the QED background is diminished by a factor of more than 70, while the Higgs signal is reduced by about $40 \% 9$

(b) The prolific gluon dijets may mimic $\tau^{+} \tau^{-}$production since one may misidentify the outgoing gluons as $\tau^{\prime}$ 's. To evaluate this contribution we use the technique of Ref. [16] to calculate the effective exclusive $g g^{P P}$ luminosity and the Born $g g^{P P} \rightarrow g g$ hard cross section. Here $g g^{P P}$ indicates that the 'active' gluons, which interact to form the system with mass $M$, originate from colourless $t$-channel exchanges, see Fig. 1, Imposing the same $\theta$ cut, $60^{\circ}<\theta<120^{\circ}$, we obtain

$$
\sigma(p p \rightarrow p \oplus g g \oplus p)=45 \mathrm{pb} \frac{2 \Delta M}{M}\left(\frac{120 \mathrm{GeV}}{M}\right)^{5} .
$$

To suppress this QCD background down to the level of $1 \mathrm{fb}$ at $M=120 \mathrm{GeV}$, the probability that a gluon is misidentified as a $\tau, P_{g / \tau}$, must be less than $1 / 50$ for each high $E_{T}$ jet 10 .

\footnotetext{
${ }^{9}$ It should be noted that since larger photon transverse momenta correspond to smaller impact parameters, the soft survival factor in the photon fusion process at $p_{T}>200 \mathrm{MeV}$ falls to $S^{2} \simeq 0.5$.

${ }^{10}$ In Ref. [76], for the inclusive case, the probability $P_{g / \tau}$ was evaluated as $1 / 500$. It therefore seems quite realistic to expect that the probability $P_{g / \tau}<1 / 50$ can be achieved in the much cleaner environment of a CED event.
} 


\subsection{The backgrounds to the $p \oplus\left(h, H \rightarrow W W^{(*)}\right) \oplus p$ signal}

The $W W^{(*)}$ decay mode of the Higgs is less challenging than the $b \bar{b}$ channel from a purely experimental perspective. As discussed in detail in Refs. [77,78], another attractive feature of the $W W^{(*)}$ channel is that in this case there is no relatively large continuum background process, such as central exclusive $b \bar{b}$ production in the case of $H \rightarrow b \bar{b}$. It should be recalled that the latter strongly relies on the experimental missing mass resolution and misidentification probability $P_{g / b}$ being sufficiently well under control to provide adequate background suppression.

Events with two $W$ bosons in the final state fall into 3 broad categories depending on the decay modes of the $W$ 's: fully-hadronic, semi-leptonic and fully-leptonic. Experimentally, events in which at least one of the $W$ 's decays in either the electron or muon channel are by far the simplest, and the analysis (see Refs. [77,78]) focuses on the semi- and fully-leptonic modes, which constitute about half the signal.

The main exclusive backgrounds in the case of the $W W$ channel can be divided into two broad groups:

1. Central production of a $W W$ pair, $p p \rightarrow p \oplus\left(W W^{(*)}\right) \oplus p$, from the process $\gamma \gamma \rightarrow$ $W W^{(*)}$.

2. The $W$-strahlung process $p p \rightarrow p \oplus W j j \oplus p$ arising from the $g g^{P P} \rightarrow W q \bar{q}$ subprocess, where the (hadronically decaying) $W^{*}$ is 'faked' by the two quarks.

As shown in Ref. [77], over a wide region of Higgs masses the photon-photon backgrounds can be strongly suppressed if one requires that the final leptons and jets are central and impose cuts on the transverse momenta of the protons in the taggers. At the same time, these cuts do not significantly affect the signal.

The potentially problematic background arises from the second category above, i.e. from the QCD $W$-strahlung processes, which have been studied in detail in Ref. [78]. Following this analysis, above the $W W$ threshold the background should not be a problem, since its contribution can be suppressed extremely effectively by requiring the invariant mass of the di-quark system to be close to $M_{W}$.

For $M_{H}<2 M_{W}$, there are two distinct scenarios in which either the $W$ or the $W^{*}$ decays leptonically. In the latter case the QCD background has been shown to be very small. For the former, without additional cuts on the final state, the calculated $g g$ background is of the same order as the SM Higgs signal. Though a full MC simulation would be required to assess the effectiveness of the further optimisation approaches, we expect that procedures such as cuts on the final state particles and azimuthal correlations between jets, should enable this background to be significantly reduced further without dramatically affecting the signal.

To summarise the current understanding, further optimisation efforts are required in order to reduce the QCD background contribution, arising in the semi-leptonic case when the offshell $W$ boson decays hadronically. For the fully leptonic decay modes, and for semi-leptonic decays in which the on-mass-shell $W$ boson decays hadronically, the signal-to-background ratio for a SM-type Higgs boson should be much greater than unity. As explained above, an important property of the $W W$ channel is that in this case the suppression of the dominant backgrounds does not rely primarily on the high precision of the missing mass resolution. 


\section{CED production of the $\mathcal{C P}$-even Higgs bosons $h$ and $H$ : experimental aspects}

In the following we will discuss the prospective experimental efficiencies for the various CED channels. As discussed above, in the MSSM the Higgs-boson phenomenology is very different from the SM case. The light $\mathcal{C P}$-even MSSM Higgs boson cannot be heavier than about $130 \mathrm{GeV}[38,39]$. For large values of $\tan \beta$ and not too large $M_{A}$, its couplings to bottom quarks and $\tau$ leptons can be strongly enhanced compared to the SM case. In certain parameter regions, on the other hand, also a strong suppression of the couplings of the light $\mathcal{C P}$-even MSSM Higgs boson to down-type fermions is possible, leading in this case to an enhancement of $\operatorname{BR}\left(h \rightarrow W W^{(*)}\right)$. Within the SM with increasing Higgs-boson mass the branching ratios of the Higgs boson into $b \bar{b}$ and $\tau^{+} \tau^{-}$fall very rapidly due to the rise of the branching ratios into the $W W^{(*)}$ and $Z Z^{(*)}$. In the MSSM, on the other hand, the $H$ boson decouples from the gauge bosons if its mass is much higher than the upper bound on the mass of the light Higgs, and the $A$ boson has zero couplings to the SM gauge bosons at tree-level. Consequently, the decays of $H, A$ into $b \bar{b}$ and $\tau^{+} \tau^{-}$remain dominant as long as no decay channels into supersymmetric particles (or light Higgs bosons) are open. Therefore in the mass region above about $130 \mathrm{GeV}$ the decay channels $H \rightarrow b \bar{b}, \tau^{+} \tau^{-}$are much more important in the MSSM as compared to the SM case. The couplings of $H, A$ to down-type fermions receive an additional enhancement in the large $\tan \beta$ region. On the other hand, the decay of the neutral $\mathcal{C} \mathcal{P}$-even MSSM Higgs bosons into $W$ bosons, $h, H \rightarrow W W^{(*)}$ is significant only for relatively low masses, i.e. $M_{h}, M_{H} \lesssim 130 \mathrm{GeV}$.

A detailed investigation of the Higgs sector is one of the central physics targets of the recent proposal [79] to add proton tagger (Roman Pot (RP)) detectors positioned at a distance $\pm 420 \mathrm{~m}$ from the interaction points of the ATLAS and CMS experiments at the LHC. At nominal LHC optics this will allow coverage in the proton fractional momentum loss $\xi$ in the range $0.002-0.02$, with an acceptance of around $30 \%$ for a centrally produced system with a mass around $120 \mathrm{GeV}$. A combination with the forseen proton detectors at $\pm 220 \mathrm{~m}[25,27]$ would significantly increase the physics reach of forward studies enlarging the $\xi$ range up to 0.2. This would be especially beneficial because of the acceptance for higher mass states and improvements in the triggering, see the discussion below and Ref. [26].

Experimentally, for increasing mass of the centrally produced system, the detector characteristics improve and the overall environment becomes cleaner. When the mass increases, the overall proton tagger acceptance (which includes all the combinations of $420 \mathrm{~m}$ and $220 \mathrm{~m}$ taggers) rises, also the trigger and $b$-tagging efficiencies increase, and the Higgs-boson mass resolution can improve. The signal-to-background ratio should also increase since it behaves roughly as $M^{3} / \Delta M$, where as before $\Delta M$ is the mass window over which the signal and background is collected, see eqs. (19), (20) and (21).

While the total RP acceptance increases with rising mass, the acceptance of the $420+420 \mathrm{~m}$ (referred to below as "420") configuration starts to decrease around a mass of $90 \mathrm{GeV}$, so that the fraction of the CED signal events detected at this configuration becomes marginal for masses above $200 \mathrm{GeV}$. In general, the low mass signal comprises events detected at $220+420 \mathrm{~m}$ or $420+220 \mathrm{~m}$ (referred to below as "combined" configuration) and in the 420 configuration, while the high-mass signal consists mainly of events detected at $220+220 \mathrm{~m}$ 
(denoted as "220") and in the combined configuration. For $M_{H} \gtrsim 250 \mathrm{GeV}$ only the $220 \mathrm{RP}$ configuration is relevant.

The mass resolution, $\delta M$, however, depends on the RP configuration. At $M=120 \mathrm{GeV}$ in the 420 case a mass resolution of $\delta M=1.9 \mathrm{GeV}$ is achievable, whereas the resolution becomes 2-3 times worse for the combined or 220 configurations. Because of worsening mass resolution, in order to collect a sufficient part of the signal we have to apply a wider mass window than in the 420 case. By enlarging the mass window, the collected signal increases as a consequence of the convolution of Gaussian and Breit-Wigner distributions (as will be explained below), while the background contribution is directly proportional to the width of the mass window. At some point, the statistical significance stops rising and starts to decrease. Therefore, it becomes essential to find the optimal mass window widths for all different RP configurations. The procedure we used to find the optimum mass window is described below.

We now briefly describe the main selection criteria for the three basic decay channels:

- $h, H \rightarrow b \bar{b}$ : In the current study the signal comprises two main event categories: (a) two $b$-tagged jets and (b) two jets with at least one $b$-hadron decaying into a muon. The signature for (a) consists of three main ingredients: $(i)$ two scattered protons, one in each arm of the RPs, $(i i)$ two well-collimated $b$-tagged jets (back-to back in the azimuthal angle $\left.\phi, 2.85<\left|\phi_{1}-\phi_{2}\right|<3.43\right)$ with rapidities $\left|\eta_{\text {jet }}\right|<2.5$ in the central detector, and (iii) consistent values of the whole 4-momentum (mass, rapidity and transverse momentum) of the central mass system as evaluated from the two tagged protons ("missing mass") and as determined from the two jets. In particular the following cuts were imposed on the longitudinal momentum fractions $x^{+}, x^{-}$and the ratio of the dijet mass $M_{b b}$ measured in the central detector to the missing mass $M_{\text {missing }}$, given by the RPs (see Ref. [26]):

$$
\begin{aligned}
\left|x_{b b}^{k}-x_{\text {missing }}^{k}\right| / x_{\text {missing }}^{k} & <0.3(\mathrm{k}=+,- \text { denote the light cone components of the } \\
\text { momenta in opposite beam directions) } & \\
0.85<M_{b b} / M_{\text {missing }} & <1.15 \text { (for the } 420 \text { configuration) } \\
0.8<M_{b b} / M_{\text {missing }} & <1.2 \text { (for the combined and } 220 \text { RP configurations) }
\end{aligned}
$$

The other event category (b) with muons in the final state yields about $10 \%$ of the whole signal sample. The signal is selected in the same way as in the event category (a) except that the jets are not required to be b-tagged and in addition, the jet plus muon trigger conditions are applied (a jet with $E_{\mathrm{T}}>40 \mathrm{GeV}$ and at least one muon with $E_{\mathrm{T}}>3 \mathrm{GeV}$ ). Further details on the selection cuts can be found in Ref. [26]11.

- $h, H \rightarrow \tau^{+} \tau^{-}$: This channel has a dominant dijet signature, but also sizable branching ratios of $\tau \rightarrow l \nu \bar{\nu}$. These features render this channel similar to the $h, H \rightarrow b \bar{b}$ channel. Therefore, we conservatively assume the same selection efficiencies as in the case of decays to $b \bar{b}$.

\footnotetext{
${ }^{11}$ It would be beneficial to include electrons originated from the $b$-decays; at the moment this is still under discussion.
} 
- $h, H \rightarrow W W^{(*)}$ : The experimental feasibility of the mode $h, H \rightarrow W W^{(*)}$ has been studied in detail in Refs. [77,78]. Triggering on this channel is not a problem, since the final state is rich in high- $p_{\mathrm{T}}$ leptons. Efficiencies of about $20 \%$ can be achieved if the standard leptonic and di-leptonic trigger thresholds are applied. It was demonstrated in Refs. $[77,78]$ that there would be a detectable signal with small and controllable background for the CED production of a SM-like Higgs boson in the mass interval between $140 \mathrm{GeV}$ and $200 \mathrm{GeV}$. Unfortunately, based on the standard lepton Level 1 trigger thresholds and Roman Pot acceptances (see Tab. 1 and Ref. [77]), the yield for a $120 \mathrm{GeV}$ SM Higgs boson with an integrated luminosity of $\mathcal{L}=60 \mathrm{fb}^{-1}$ is only about 3 events 12 . The prospects become better for higher luminosity. Furthermore, as we will discuss below, the potential of the $h, H \rightarrow W W^{(*)}$ mode may improve in favourable regions of the MSSM parameter space when the decay $h \rightarrow b \bar{b}$ is suppressed [49].

For the purpose of this paper we define the overall efficiency, $\epsilon$, which incorporates the RP and central detector acceptances, experimental cuts, efficiencies of the central detector and Level 1 triggers. In the $h, H \rightarrow b \bar{b}$ case it also accounts for the $b$-tagging efficiency and for the cut on the rapidities of the central jets $\left|\eta_{\text {jet } 1}-\eta_{\text {jet } 2}\right|<1.113$. The same angular (rapidity) cut is imposed in the $\tau \tau$ channel in order to suppress the QED background. The parameter $\epsilon$ relates the cross section that can actually be detected, $\sigma_{\text {det }}$, to the theoretical cross section $\sigma_{\text {th }}$ (calculated using eqs. (19), (20) and (21)), which would correspond to the case that no experimental cuts are applied and no efficiency losses occur. Accordingly,

$$
\sigma_{\mathrm{det}}=\epsilon \cdot \sigma_{\mathrm{th}}
$$

The background processes relevant for the different CED channels have been discussed in Sects. 4.14.3. To retain the signal at the Level 1 trigger, the following trigger conditions can be used:

1. Single-sided 220 $m R P$ and at least two jets, each with $E_{\mathrm{T}}>40 \mathrm{GeV}$, measured in the central detector.

As was shown in Ref. [80], for this trigger a tolerable Level 1 bandwidth of $1 \mathrm{kHz}$ per experiment may be kept only up to luminosities of about $\mathcal{L} \sim 2 \times 10^{33} \mathrm{~cm}^{-2} \mathrm{~s}^{-1}$ due to pile-up background. Requiring the single-sided $220 \mathrm{~m} \mathrm{RP}$ condition implies that the symmetric $420 \mathrm{RP}$ configuration may only be possible for the events where the acceptances of $220 \mathrm{~m}$ and $420 \mathrm{~m}$ RPs at the same side overlap. In such events (amounting roughly to $40 \%$ of the total event yield for the $b \bar{b}$ decay mode at a mass of $120 \mathrm{GeV}$ in the CMS-TOTEM system) the information from $420 \mathrm{~m}$ is going to be used due to a better resolution on the fractional momentum loss $\xi$ of the proton. In the rest of the signal retained by this Level 1 trigger condition, the combined RP configuration will be present resulting in a 2-3 times worse mass resolution than for the 420 case (see above).

12 The yield would rise if the lepton Level 1 leptonic trigger thresholds could be reduced [77].

13 In the configuration with two forward outgoing protons this is equivalent to the cut $60^{\circ}<\theta<120^{\circ}$ on the polar angle of $b$-jets in the $b \bar{b}$ rest frame, required to reduce the QCD background (see the discussion in Sect. 4.1). 
The efficiency of this trigger to retain that part of the signal which passes the cuts on the two jets and does not include selected muons rises from about $60 \%$ at $M=120 \mathrm{GeV}$ to $100 \%$ at $M=200 \mathrm{GeV}$.

2. A jet with $E_{\mathrm{T}}>40 \mathrm{GeV}$ and at least one muon with $E_{\mathrm{T}}>3 \mathrm{GeV}$, both measured in the central detector.

This trigger retains that part of the signal that comes from the decays of $b$-hadrons or $\tau$ leptons into muons. Therefore it also increases the fraction of the $420 \mathrm{RP}$ configuration in the total signal sample. The saving efficiency for the signal events of the above type that pass the cuts is $100 \%$ for all masses.

3. At least two jets each with $E_{\mathrm{T}}>90 \mathrm{GeV}$ measured in the central detector.

This is the lowest $E_{\mathrm{T}}$ threshold for any dijet trigger designed at ATLAS or CMS. Obviously, all the signal with masses well above $200 \mathrm{GeV}$ (and passing the cuts) may be retained at Level 1 just by this trigger.

4. Leptonic triggers, requiring electrons or muons in the central detector.

This trigger serves mainly to retain events with $W$ bosons decaying leptonically or semileptonically. In CMS, the $E_{\mathrm{T}}$ thresholds are 29, 10, 14 and $3 \mathrm{GeV}$ for the single-electron, double-electron, single-muon and double-muon triggers, respectively. Corresponding values for ATLAS are 25, 15, 20 and $10 \mathrm{GeV}$, but the trigger efficiencies are similar.

For the process $h, H \rightarrow b \bar{b}$, a combination of the triggers 1 and 2 allows the retention of about $65 \%$ of the signal events passing the relevant cuts at $M=120 \mathrm{GeV}$ and up to $100 \%$ at $M=200 \mathrm{GeV}$, while at masses well above $200 \mathrm{GeV}$ the trigger 3 retains the whole signal sample selected by the cuts. On the other hand, the signal for the process $h, H \rightarrow W W^{(*)}$ (with the leptonic decay of at least one $W$ ) is collected by applying the cuts corresponding to the trigger definition only, without any additional selections.

\begin{tabular}{||c||c|c|c|c|c|c|c||}
\hline \hline$M[\mathrm{GeV}]$ & $A_{420}$ & $A_{\text {comb }}$ & $A_{220}$ & $\epsilon_{420}(b \bar{b})$ & $\epsilon_{\text {comb }}(b \bar{b})$ & $\epsilon_{220}(b \bar{b})$ & $\epsilon\left(W W^{(*)}\right)$ \\
\hline \hline 100 & 37 & 13 & 0 & 1.2 & 0.8 & 0 & - \\
\hline 120 & 31 & 25 & 0 & 1.7 & 2.5 & 0 & 11.7 \\
\hline 140 & 25 & 37 & 0 & 1.6 & 5.1 & 0 & 14.6 \\
\hline 160 & 19 & 49 & 0 & 1.5 & 7.6 & 0 & 20.4 \\
\hline 180 & 14 & 60 & 0 & 1.2 & 9.6 & 0 & 21.5 \\
\hline 200 & 9 & 69 & 0 & 0.4 & 11.0 & 0 & 24.2 \\
\hline 300 & 0 & 76 & 13 & 0 & 12.5 & 2.0 & - \\
\hline
\end{tabular}

Table 1: RP acceptances $(A)$ for the 420, combined and $220 \mathrm{RP}$ configurations, and total experimental selection efficiencies for the signal decay channels $h, H \rightarrow b \bar{b}$ and $h, H \rightarrow$ $W W^{(*)}$, evaluated under the assumption that the whole signal is collected at a given mass, see also Ref. [26]. All numbers are given in \%. They have been obtained with ExHuME 1.3 [63]. Conservatively, $\epsilon\left(h, H \rightarrow \tau^{+} \tau^{-}\right)$is set equal to $\epsilon(h, H \rightarrow b \bar{b})$ (with $\epsilon$ defined in eq. (25)). 
Tab. 1 shows RP acceptances as a function of the Higgs-boson mass for all possible RP configurations. We also show the signal selection efficiencies that have been used in our evaluation of the statistical significances. Following Ref. [4], the statistical significance has been calculated as a probability from the Poisson distribution with mean equal to the number of background events to observe a number of events equal or greater than a sum of signal and background events, converted to an equivalent number of sigmas of Gaussian distribution [81]. The number of signal events at a given mass has been obtained by scaling the signal cross section from eq. (19) or (20) by the signal selection efficiencies from Tab. 1). The same procedure has been applied to obtain the number of background events with the cross section calculated using eq. (21). In the calculation of the significance, we considered the number of signal and background events without systematic uncertainties. As mentioned above, both the signal and the background consist of two contributions corresponding to the two RP configurations, namely 420 and combined up to masses of $200 \mathrm{GeV}$, and 220 and combined for masses above $200 \mathrm{GeV}$.

In the case of large $\tan \beta$ the total width $\left(\Gamma_{\text {tot }}\right)$ of the Higgs bosons may become rather large. Therefore, in what follows we choose the optimal mass window

$$
\Delta M(M, \tan \beta)=2 \sqrt{\delta M^{2}(M)+\Gamma_{\text {tot }}^{2}(M, \tan \beta)},
$$

where the mass resolution $\delta M$ was taken from the RP studies made in Ref. [32]. The values of the mass window range from 3 to $20 \mathrm{GeV}$ for the 420 case and between 11 and $22 \mathrm{GeV}$ for the combined or $220 \mathrm{RP}$ configurations. The lower boundaries correspond to low values of $\Gamma_{\text {tot }}$, while the larger boundaries correspond to larger values of $\Gamma_{\text {tot }}$.

In our analysis we use the optimum mass window as the region over which the signal as well as background cross sections are integrated to obtain the numbers of signal $(S)$ and background $(B)$ events:

$$
\begin{aligned}
S & =\sigma^{S}(M) \mathcal{L}\left[\mathcal{I}_{420}(M, \tan \beta) \epsilon_{420}(M)+\mathcal{I}_{\mathrm{comb}}(M, \tan \beta) \epsilon_{\mathrm{comb}}(M)\right], \\
B & =\frac{\mathrm{d} \sigma^{B}}{\mathrm{~d} M} \mathcal{L}\left[\Delta M_{420}(M, \tan \beta) \epsilon_{420}(M)+\Delta M_{\mathrm{comb}}(M, \tan \beta) \epsilon_{\mathrm{comb}}(M)\right],
\end{aligned}
$$

where $\sigma^{S}$ and $\mathrm{d} \sigma^{B} / \mathrm{d} M$ are the cross sections calculated using eqs. (19), (20) and (21) 14 . $\mathcal{I}_{420}$ and $\mathcal{I}_{\text {comb }}$ are the integrals of convolutions of Gaussian and Breit-Wigner mass distributions over a given mass window for the 420 and combined RP configuration, respectively, and $\Delta M_{420}$ and $\Delta M_{\text {comb }}$ are the corresponding mass windows. Since the total width, which depends on $\tan \beta$, enters $\Delta M_{420}$ and $\Delta M_{\text {comb }}$, our background rate given in eq. (28) formally depends on $\tan \beta$. It should be noted that in a situation where the total width is larger than the mass resolution, the CED process may provide a unique opportunity to actually measure the total width. This information may be very important for distinguishing an MSSM Higgs boson from a SM-type Higgs.

Because of the acceptance, the $220 \mathrm{RP}$ configuration only contributes for the highest mass value given in Tab. 1, i.e. $M=300 \mathrm{GeV}$. Because of similar mass resolutions for

\footnotetext{
${ }^{14}$ When calculating $B$, we actually integrated over the mass window from $M-\Delta M / 2$ up to $M+\Delta M / 2$ explicitly. We obtained the same result as using eq. (28) which is due to the fact that the effect of the first derivative with respect to $M$ is cancelled by the integration over the symmetric interval, and only the second derivative may contribute.
} 
the combined and $220 \mathrm{RP}$ configurations, in this mass region contributions of both the RP configurations are summed and denoted by "combined". When calculating the integrals $\mathcal{I}$, we account for the mass dependence of the hard subprocess, the experimental efficiencies (from Table 1) and the effective exclusive $g g^{P P}$ luminosity. For this particular choice of the optimum mass window, the values of $\mathcal{I}_{420}$ and $\mathcal{I}_{\text {comb }}$ are shown in Table 2 . Evidently they are close to 0.67 over the whole studied range of the values of the mass and the total width. As a systematic cross-check, we have also tried another option for the optimum mass window (arising from a more complete calculation), namely $\Delta M=\sqrt{(2.7 \delta M)^{2}+\left(1.5 \Gamma_{\text {tot }}\right)^{2}}$, and observed very similar results as for the previous option.

\begin{tabular}{||c||c|c|c|c|c||}
\hline$M[\mathrm{GeV}]$ & $\Gamma_{\text {tot }}=0.2$ & 1 & 5 & 10 & $20[\mathrm{GeV}]$ \\
\hline \hline 120 & $65 / 67$ & $62 / 66$ & $69 / 66$ & $71 / 71$ & $75 / 80$ \\
\hline 140 & $64 / 67$ & $61 / 65$ & $69 / 63$ & $69 / 65$ & $70 / 67$ \\
\hline 160 & $62 / 67$ & $62 / 65$ & $69 / 62$ & $70 / 66$ & $71 / 68$ \\
\hline 180 & $62 / 67$ & $62 / 64$ & $67 / 64$ & $67 / 67$ & $68 / 69$ \\
\hline 200 & $61 / 67$ & $59 / 64$ & $62 / 63$ & $65 / 67$ & $83 / 69$ \\
\hline 300 & $-/ 68$ & $-/ 65$ & $-/ 63$ & $-/ 67$ & $-/ 70$ \\
\hline
\end{tabular}

Table 2: The values of the integrals $\mathcal{I}_{420} / \mathcal{I}_{\text {comb }}$ [in \%] for the 420 and combined RP configurations.

For the $h, H \rightarrow \tau^{+} \tau^{-}$sample it may be possible to exploit the full $420 \mathrm{RP}$ information in the off-line analysis. Using information on the event topology and other characteristics of the $h, H \rightarrow \tau^{+} \tau^{-}$sample, it may be possible to avoid the single-sided $220 \mathrm{~m}$ RP condition for the $\tau^{+} \tau^{-}$final state. However, no detailed studies of the trigger for such events have been performed so far [82].

From the discussion above, it is evident that it would be beneficial if the $420 \mathrm{~m} \mathrm{RPs}$ could be incorporated into the Level 1 trigger. Based on the currently foreseen hardware this seems not to be feasible, given the scope of the Level 1 trigger buffer and the time that the signal needs to travel from the $420 \mathrm{~m} \mathrm{RP}$ detector. An increase of the Level 1 trigger latency would allow one to use the $420 \mathrm{~m}$ RP information for triggering at Level 1 [83]. Such a setup could be very advantageous for suppressing pile-up and non-pile-up backgrounds. It is worth noting in this context that an increase of the Level 1 trigger latency, as needed for triggering on the $420 \mathrm{~m} \mathrm{RP}$ information, has been discussed in the context of the LHC luminosity upgrade (SLHC) [84].

In the above discussion of the experimental prospects for CED Higgs production we have particularly emphasised the importance of triggering in the central detector on leptons arising from $b, \tau$ or $W$ decays. From the experimental point of view, in order to further enhance the physics potential of the CED Higgs processes an improvement of the signal selection efficiency by lowering the lepton thresholds would therefore be beneficial.

Bearing in mind the remaining theoretical uncertainties on the signal rates (e.g. from higher-order QCD effects), the results for the signal rates in this study represent a conservative evaluation. A possible enhancement of the signal rates by a factor of about 2 would still 
be compatible with our estimates of the theoretical uncertainties. Furthermore, on the experimental side a gain in sensitivity might be expected from improvements and optimisation of the event selection procedures, triggers and various efficiencies. Besides the above-mentioned possibility of including the $420 \mathrm{RP}$ into the Level 1 trigger, one could envisage improvements from lowering the lepton $p_{\mathrm{T}}$ thresholds in the existing Level 1 triggers, from considering, possibly, an electron trigger and from improving the selection procedure for the $b \bar{b}$ and $\tau \tau$ signals. In order to illustrate the physics gain that could be expected if such a more optimistic scenario were to be realised, we will include in the following a discussion of the cross sections and experimental efficiencies outlined above with scenarios where the event rates are higher by a factor of 2 (due to improvements on the experimental side and possibly higher signal rates, denoted by "eff $\times 2$ ").

Finally, it is important to recall that the price to pay for the increase of the LHC luminosity is a rise in the average number, $N$, of soft proton-proton interactions per bunch crossing. Thus, at an instantaneous luminosity of $2 \times 10^{33} \mathrm{~cm}^{-2} \mathrm{~s}^{-1} N=7$, while at $10^{34} \mathrm{~cm}^{-2} \mathrm{~s}^{-1} N=35$. In such a situation each hard scale central event 15 is accompanied by a luminosity-dependent number of minimum bias events, which dominantly are of soft origin. A certain fraction of these pile-up events contains protons within the acceptances of the RPs. In particular, from an evaluation based on Ref. [85] it follows that about 2-3\% (0.75-1\%) of the minimum bias events can be detected in the RPs at $220 \mathrm{~m}(420 \mathrm{~m})$ on one side. When the same $\mathrm{RP}$ acceptances are applied, these numbers appear to be in a reasonable agreement with the results of Ref. [26], where Phojet [86] was used to generate minimum bias events. The pile-up events could potentially endanger the prospects of CED studies at high luminosities, since they can be overlaid with hard scale inclusive non-diffractive events. None of these events would separately survive the signal selection cuts, but once two single diffractive events, each with a proton within the RP acceptance, are overlaid with a hard scale central signal, this mixture of three events may perfectly fake the signal. The problem is that the rates of inclusive processes are so huge compared to the CED signal that this situation may occur quite frequently. As a result, event pile-up is (currently) considered to be one of the most important background sources in the planned high instantaneous luminosity runs.

The pile-up issue is currently under very intensive study within ATLAS and CMS (for a detailed discussion see Ref. [26]). Although at first sight the issue of pile-up backgrounds does not look favourable, the situation is far from being hopeless. Apart from concentrating on the $420 \mathrm{~m}$ RP configuration, which enables a narrow mass window to be imposed, there are other possible leverages that could bring the pile-up problem under control.

In the FP420 project [79] there are prospects for installing fast timing detectors with an expected vertex resolution of better than $3 \mathrm{~mm}$. Such a precision would enable one to determine whether the protons seen in the RPs came from the same vertex as the hard scale central signal. Preliminary Monte Carlo studies indicate that, with the nominal LHC running conditions, a rejection of a factor of about 40 should be possible [27].

The pile-up effect can further be reduced by exploiting precise vertex detectors with vertex resolution of better than $100 \mu \mathrm{m}$ : events with more than one vertex in a few $\mathrm{mm}$ window in the beam direction given by the timing detectors (presumably from pile-up) can then be rejected [26]. Another possibility to reduce the effect of pile-up is to exploit different

\footnotetext{
${ }^{15}$ Here "hard scale event" refers to an event with a large $E_{\mathrm{T}}$ jet (or lepton), or where a particle with a large mass (for example, a $W$-boson) is produced.
} 
track multiplicity properties of signal and pile-up events. A cut reflecting this difference may provide another rejection factor of up to 100 [87]. Here, however, further studies have to be performed, in particular with full detector simulations.

Finally, matching the whole 4-momentum (not just the mass, but also the rapidity and transverse momenta) of the central heavy system (dijet, $W W^{(*)}$-pair) measured in the central detector to the corresponding values coming from the forward protons taggers will further suppress the pile-up background.

It should be noted that the situation concerning pile-up backgrounds is more favourable in the $W W^{(*)}$ channel compared to the channel with a $b \bar{b}$ pair in the central detector. Indeed, in the SM case the signals expected in the $b \bar{b}$ and $W W^{(*)}$ channels are of the same order of magnitude. The lower probability for the light Higgs to decay to $W W^{(*)}$ is partly compensated by a better efficiency of the $W$ signal selection. On the other hand, the probability of $W$ production in the pile-up event is lower than the probability of high $E_{\mathrm{T}} b$-jet production. In fact the suppression caused by the large mass $\left(M_{W} \sim 80 \mathrm{GeV}\right)$ of a $W$ boson is slightly stronger than that caused by the large $E_{\mathrm{T}} \sim 40-50 \mathrm{GeV}$ of a $b$-quark jet. Besides this, the electroweak coupling, which controls the $W$ production rate, is smaller than the QCD coupling $\alpha_{s}$. As a result, the signal-to-background ratio $S / B_{\text {pile-up }}$, where $B_{\text {pile-up }}$ is the the pile-up background, is expected to be higher by around two orders of magnitude for the $W W^{(*)}$ channel as compared to the $b \bar{b}$ case.

Concerning triggering at Level 1, one may hope that the pile-up background contaminating the Level 1 trigger output rate may be managed even at instantaneous luminosities higher than $2 \times 10^{33} \mathrm{~cm}^{-2} \mathrm{~s}^{-1}$ (which was found in initial studies [80] to be the highest luminosity compatible with a tolerable Level 1 bandwidth of $1 \mathrm{kHz}$ per experiment). In particular, if a Higgs boson has already been detected in the standard (non-diffractive) search channels, the knowledge about its mass can be embedded into the proposed diffraction Level 1 trigger. Thus this information can be used to significantly suppress the pile-up background (by restricting $\xi$ values accepted at Level 1). Another option would be to incorporate the information from fast timing detectors into the Level 1 trigger logic.

In conclusion, we believe that it is conceivable that with intensive studies ATLAS and CMS will succeed in bringing the effect of pile-up down to a tolerable level even at the highest luminosities which will be delivered after several years of LHC running. In other words, even at the highest instantaneous luminosities it may be possible to select and study exclusive (CED) events in the presence of pile-up interactions.

Based on the above discussion, in our numerical analysis below we consider four scenarios for the achievable luminosities and the experimental conditions for CED processes at the LHC:

- $\underline{60 \mathrm{fb}^{-1} \text { : }}$

An integrated LHC luminosity of $\mathcal{L}=2 \times 30 \mathrm{fb}^{-1}$, corresponding roughly to three years of running at an instantaneous luminosity $\mathcal{L} \sim 10^{33} \mathrm{~cm}^{-2} \mathrm{~s}^{-1}$ by both ATLAS and CMS. With such a luminosity the effect of pile-up is not negligible but can be safely kept under control. The signal selection efficiencies are based on Tab. 1 (and are correspondingly reduced by taking the optimal mass windows into account). 
- $60 \mathrm{fb}^{-1}$ eff $\times 2$ :

The same integrated LHC luminosity as in the above scenario but with event rates that are higher by a factor of 2 (see the discussion of possible improvements and theoretical uncertainties above).

- $\underline{600 \mathrm{fb}^{-1}}$ :

An integrated LHC luminosity of $\mathcal{L}=2 \times 300 \mathrm{fb}^{-1}$ and the same efficiency factors as in the scenario with $\mathcal{L}=60 \mathrm{fb}^{-1}$. This corresponds roughly to three years of running at an instantaneous luminosity $\mathcal{L} \approx 10^{34} \mathrm{~cm}^{-2} \mathrm{~s}^{-1}$ by both ATLAS and CMS.

- $600 \mathrm{fb}^{-1}$ eff $\times 2$ :

The same integrated LHC luminosity as in the scenario with $\mathcal{L}=2 \times 300 \mathrm{fb}^{-1}$ but with event rates that are higher by a factor of 2 .

\section{Discovery reach for neutral $\mathcal{C P}$-even Higgs bosons in the MSSM in CED production}

In this section we discuss the prospects for observing the neutral $\mathcal{C} \mathcal{P}$-even MSSM Higgs bosons in CED production. We display our results in terms of the lowest-order parameters of the MSSM Higgs sector, $M_{A}$ and $\tan \beta$, for the benchmark scenarios described in Sect. 2.3. Also shown in the plots as dark shaded (blue) areas are the parameter regions excluded by the LEP Higgs searches in the channel $e^{+} e^{-} \rightarrow Z^{*} \rightarrow Z h, H[51,52]$.

For each point in the parameter space we have evaluated the relevant Higgs production cross section, see Sect. 3, times the Higgs branching ratio corresponding to the decay mode under investigation. The Higgs-boson masses, the decay branching ratios and the effective couplings for the production cross sections have been calculated with the program FeynHiggs [37-40]. The resulting theoretical cross section has been multiplied by the experimental efficiencies taking into account detector acceptances, experimental cuts and triggers as discussed in Sect. 5. The backgrounds have been estimated according to Sect. 4 .

This procedure has been carried out for four different assumptions on the luminosity scenario, see Sect. 5, and the $5 \sigma$ discovery contours (and contours for $3 \sigma$ significances, see below) have been obtained as described above.

\subsection{Prospective sensitivities for $\mathrm{CED}$ production of the light $\mathcal{C P}$ - even Higgs boson}

We start our analysis with the production of the lighter $\mathcal{C} \mathcal{P}$-even Higgs boson, $h$, and its decay into bottom quarks. As explained in Sect. 2, the $h b \bar{b}$ coupling can be significantly enhanced compared to the SM case in the region of relatively small $M_{A}$ and large $\tan \beta$ (while the lighter $\mathcal{C P}$-even Higgs boson of the MSSM behaves like the SM Higgs in the decoupling region, $M_{A} \gg M_{Z}$ ). CED production of the lighter $\mathcal{C P}$-even Higgs boson of the MSSM with subsequent decay to $b \bar{b}$ therefore yields a higher event rate in this parameter region compared to the SM case. This is shown in Fig. 2 where the ratio of signal events for the MSSM to those for the SM (with $M_{H^{\mathrm{SM}}}=M_{h}$ ) is displayed in the $M_{A}$-tan $\beta$ plane for the $M_{h}^{\max }$ (upper 

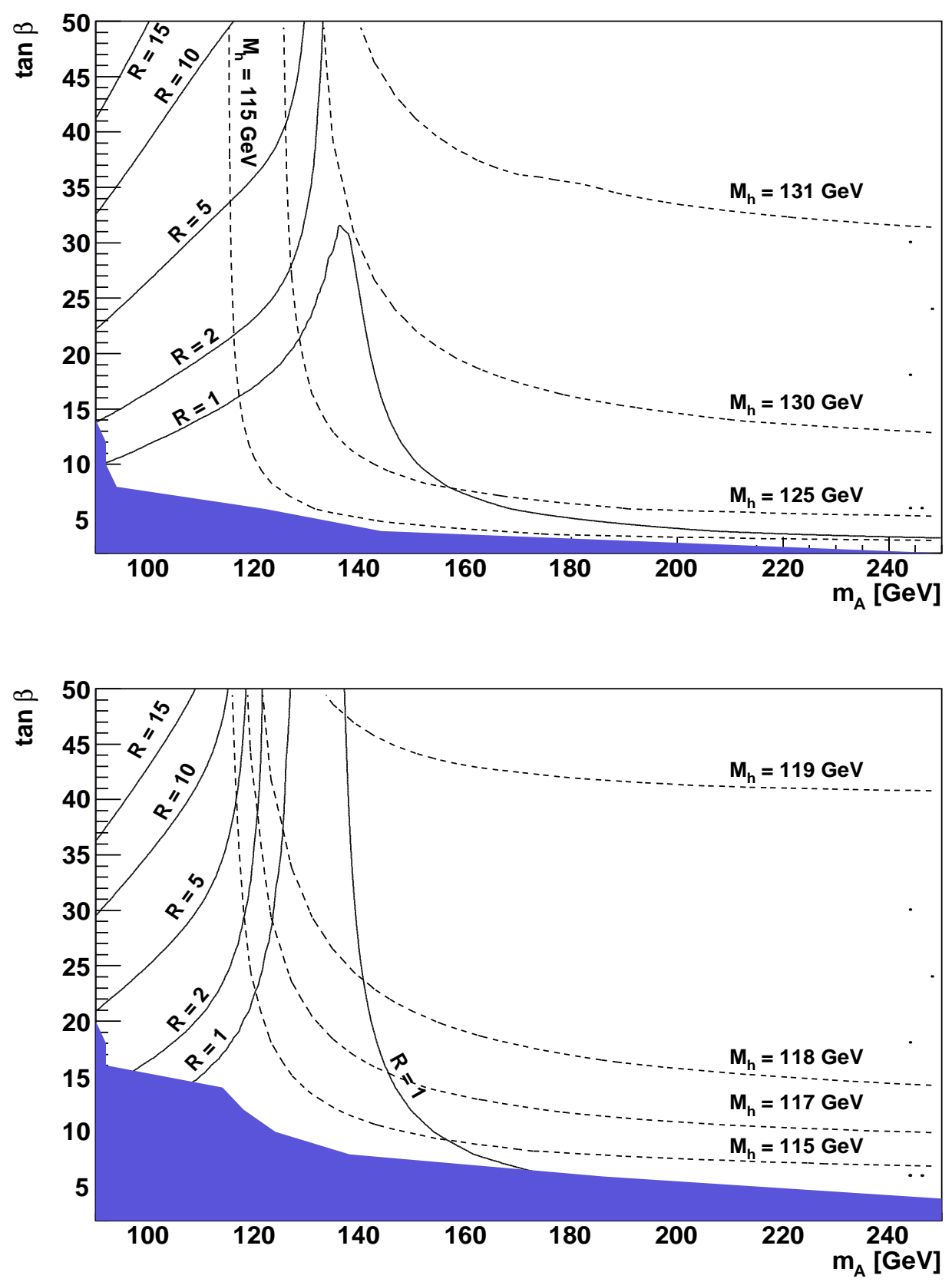

Figure 2: Contours for the ratio of signal events in the MSSM to those in the SM in the $h \rightarrow b \bar{b}$ channel in CED production in the $M_{A}-\tan \beta$ plane. The ratio is shown in the $M_{h}^{\max }$ benchmark scenario (with $\mu=+200 \mathrm{GeV}$, upper plot) and in the no-mixing scenario (with $\mu=+200 \mathrm{GeV}$, lower plot). The values of the mass of the light $\mathcal{C P}$-even Higgs boson, $M_{h}$, are indicated by dashed contour lines. The dark shaded (blue) region corresponds to the parameter region that is excluded by the LEP Higgs searches in the channel $e^{+} e^{-} \rightarrow Z^{*} \rightarrow Z h, H[51,52]$. 
plot) and no-mixing (lower plot) benchmark scenarios as specified in eqs. (12), (13). For illustration, contour lines for the mass of the lighter $\mathcal{C} \mathcal{P}$-even Higgs boson are also given. The dark shaded (blue) region indicates the part of the $M_{A}-\tan \beta$ plane that is excluded by the LEP Higgs searches in the channel $e^{+} e^{-} \rightarrow Z^{*} \rightarrow Z h, H[51,52]$. As discussed above, the exclusion bounds from the Higgs search at the Tevatron so far do not impose constraints on the parameter region with $M_{A} \gtrsim 100 \mathrm{GeV}$ and $\tan \beta \lesssim 50$.

Fig. 2 shows that the signal rate can be enhanced by up to a factor of $R=15$ in the region of large $\tan \beta$ and relatively small $M_{A}$. An enhancement by a factor of $R=5$ is possible for $\tan \beta$ values down to about $\tan \beta \approx 25$. The enhancement in the region of large $\tan \beta$ and small $M_{A}$ is slightly more pronounced in the no-mixing scenario (lower plot). This behaviour can easily be understood from the discussion in Sect. 2.2. For the value of $\mu$ chosen in the $M_{h}^{\max }$ and no-mixing scenarios, $\mu=+200 \mathrm{GeV}$, the higher-order contribution $\Delta_{b}$ is positive and therefore leads to a (slight) suppression of the bottom Yukawa coupling. Since the numerical value of $\Delta_{b}$ in the $M_{h}^{\max }$ scenario is larger than in the no-mixing scenario, as a consequence of the second term in eq. (9), the $M_{h}^{\max }$ scenario yields slightly smaller enhancement factors compared to the no-mixing scenario in the region of large $\tan \beta$ and small $M_{A}$. In contrast, the differences between the two scenarios in the parameters of the scalar top sector entering via higher-order corrections (affecting in particular the predicted value of $M_{h}$ ) give rise to a possible sizable enhancement in the $M_{h}^{\max }$ scenario up to higher values of $M_{A}$ compared to the no-mixing scenario. While an enhancement by a factor of $R=2$ occurs within the $M_{h}^{\max }$ scenario up to $M_{A} \lesssim 130 \mathrm{GeV}$, the corresponding contour is shifted towards lower $M_{A}$ values in the no-mixing scenario by about $\Delta M_{A}=10 \mathrm{GeV}$. For $M_{A} \gtrsim 150 \mathrm{GeV}$, on the other hand, the signal rates are close to the SM case in both scenarios over practically the whole range of $\tan \beta$ values, as expected from the discussion of the decoupling limit given above.

The $5 \sigma$ discovery contours in the $M_{A}-\tan \beta$ plane obtained for the four luminosity scenarios specified above are given for the $M_{h}^{\max }$ and no-mixing benchmark scenarios (i.e. the same parameters as in Fig. 2) in the upper plots of Figs. 3 and 4, respectively. The shapes of the $5 \sigma$ discovery contours in the two benchmark scenarios follow the patterns discussed in Fig. 2, the region of high $\tan \beta$ and low $M_{A}$ can be covered with slightly lower integrated luminosity in the no-mixing scenario than in the $M_{h}^{\max }$ scenario. While in the "60 $\mathrm{fb}^{-1}$ " scenario $\tan \beta$ values down to about $\tan \beta=40$ can be covered with $5 \sigma$ significance in the low $M_{A}$ region of the $M_{h}^{\max }$ scenario, the coverage in the no-mixing scenario extends to about $\tan \beta=35$ with the same luminosity (this would improve by $\Delta \tan \beta \approx 5-10$ in the more optimistic "60 $\mathrm{fb}^{-1}$ eff $\times 2$ " scenario). On the other hand, the coverage in the $M_{h}^{\max }$ scenario extends to somewhat higher $M_{A}$ values. With $600 \mathrm{fb}^{-1}$ the parameter region with $M_{A} \lesssim 135(125) \mathrm{GeV}$ and $\tan \beta \gtrsim 20$ can be covered in the $M_{h}^{\max }$ (no-mixing) scenario at the $5 \sigma$ level (and a slightly better coverage can be achieved in the more optimistic "600 $\mathrm{fb}^{-1}$ eff $\times 2$ " scenario). In the $M_{h}^{\max }$ and no-mixing scenarios the parameter $\mu$ is set to $\mu=+200 \mathrm{GeV}$. It follows from the discussion of the $\Delta_{b}$ corrections given above that the discovery reach would increase for negative values of $\mu$. We will give an example below for the case of the heavy $\mathcal{C} \mathcal{P}$-even Higgs boson. As an example of expected event rates, for $60 \mathrm{fb}^{-1}$ one would expect after all cuts in the $M_{h}^{\max }$ scenario with $\tan \beta=50$ and $M_{h} \approx 120 \mathrm{GeV}$ about 32 signal events and 28 background events, while in the no-mixing scenario with $\tan \beta=50$ and $M_{h} \approx 113 \mathrm{GeV}$ one would expect 41 signal events and 35 background events. 

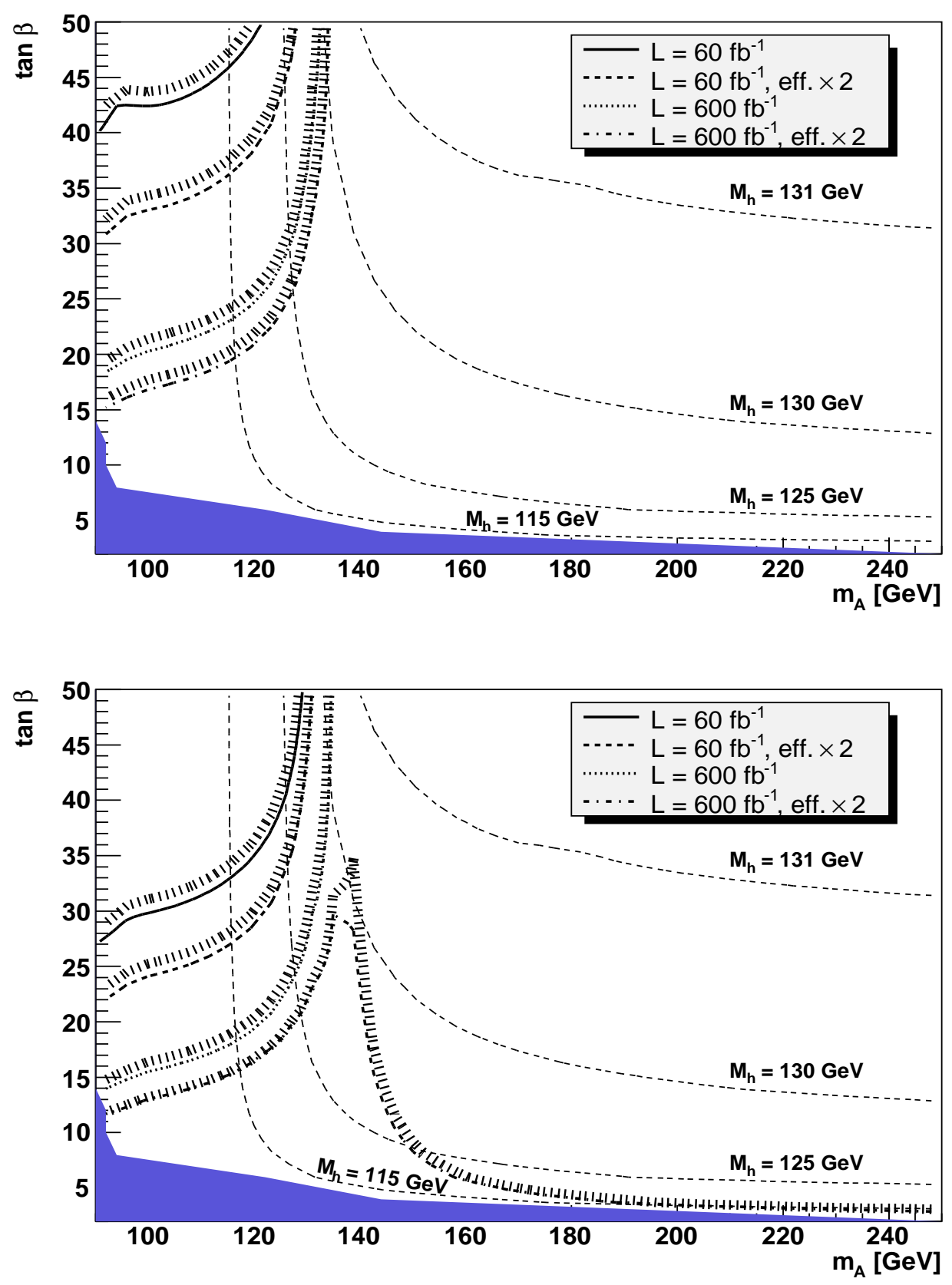

Figure 3: $5 \sigma$ discovery contours (upper plot) and contours of $3 \sigma$ statistical significance (lower plot) for the $h \rightarrow b \bar{b}$ channel in CED production in the $M_{A}-\tan \beta$ plane of the MSSM within the $M_{h}^{\max }$ benchmark scenario. The results are shown for assumed effective luminosities (see text, combining ATLAS and CMS) of $60 \mathrm{fb}^{-1}, 60 \mathrm{fb}^{-1} \mathrm{eff} \times 2,600 \mathrm{fb}^{-1}$ and $600 \mathrm{fb}^{-1} \mathrm{eff} \times 2$. The values of the mass of the light $\mathcal{C} \mathcal{P}$-even Higgs boson, $M_{h}$, are indicated by contour lines. The dark shaded (blue) region corresponds to the parameter region that is excluded by the LEP Higgs searches in the channel $e^{+} e^{-} \rightarrow Z^{*} \rightarrow Z h, H[51,52]$. 

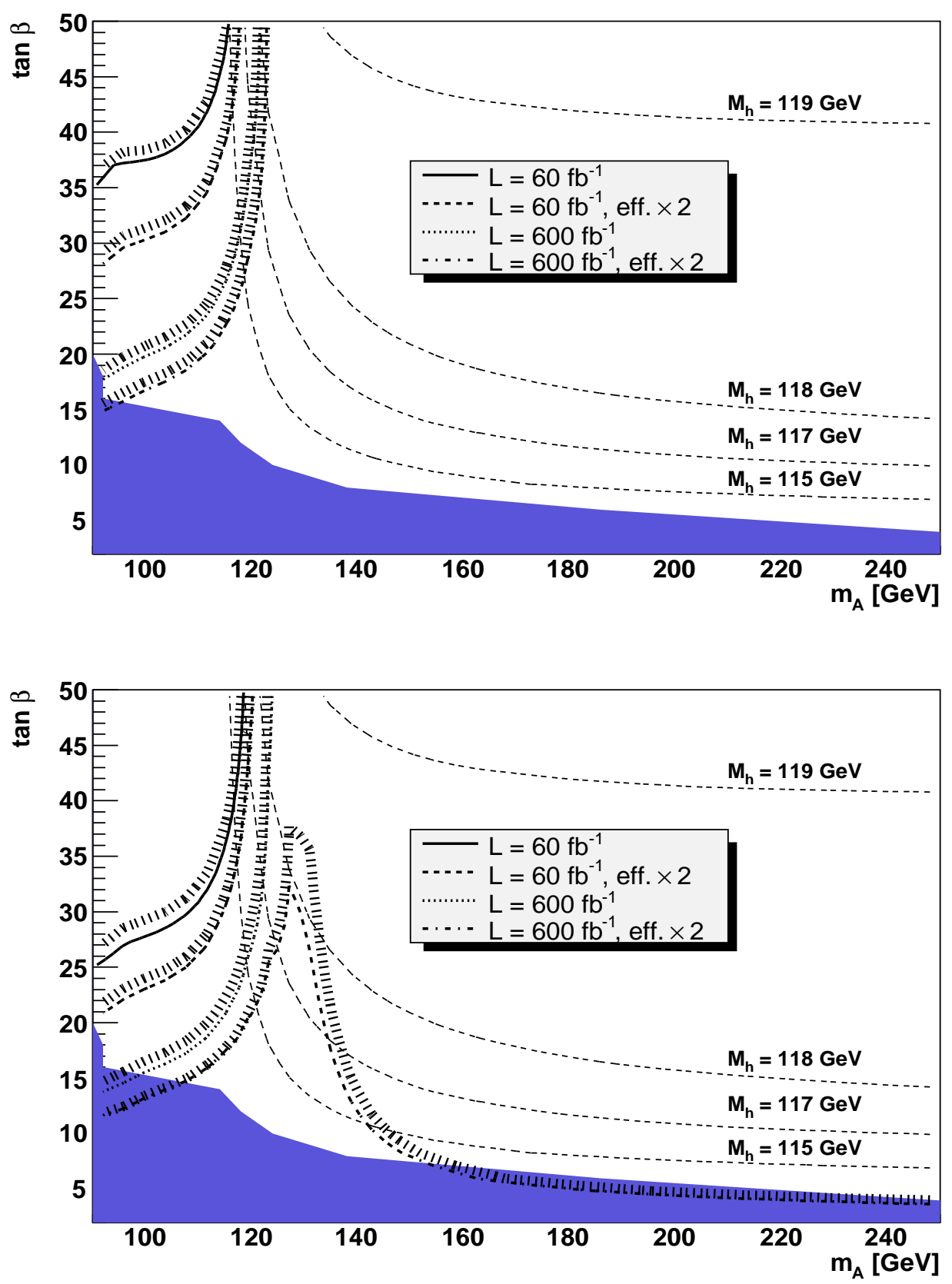

Figure 4: $5 \sigma$ discovery contours (upper plot) and contours of $3 \sigma$ statistical significance (lower plot) for the $h \rightarrow b \bar{b}$ channel in CED production in the $M_{A}-\tan \beta$ plane of the MSSM within the no-mixing benchmark scenario. The results are shown for assumed effective luminosities (see text, combining ATLAS and CMS) of $60 \mathrm{fb}^{-1}, 60 \mathrm{fb}^{-1}$ eff $\times 2,600 \mathrm{fb}^{-1}$ and $600 \mathrm{fb}^{-1}$ eff $\times 2$. The values of the mass of the light $\mathcal{C} \mathcal{P}$-even Higgs boson, $M_{h}$, are indicated by contour lines. The dark shaded (blue) region corresponds to the parameter region that is excluded by the LEP Higgs searches in the channel $e^{+} e^{-} \rightarrow Z^{*} \rightarrow Z h, H[51,52]$. 
In all our considered luminosity scenarios, a $5 \sigma$ significance for the production of the lighter $\mathcal{C P}$-even Higgs boson of the MSSM and its decay into bottom quarks is found only in the region of relatively small $M_{A}$ and large $\tan \beta$ in Figs. 3 and 4 . This is related to the fact that for a SM Higgs in the mass range corresponding to Figs. 3, 4 the statistical significance remains below the $5 \sigma$ level. Because of the enhanced signal rate in the respective part of the MSSM parameter space, as discussed above, a $5 \sigma$ discovery region occurs in the MSSM while it is absent in the SM case.

Since the lighter $\mathcal{C P}$-even Higgs boson of the MSSM is likely to be detectable also in "conventional" Higgs search channels at the LHC (see for example Refs. [1,4]), it may not be necessary to require a statistical significance as high as $5 \sigma$ for the CED channel. For illustration, we therefore also show the contours of $3 \sigma$ statistical significances, given in the lower plots of Figs. 3 and 4. Higgs production in the CED channel will provide unique information on the Higgs-boson properties. In particular, it will be important for determining the $\mathcal{C P}$ quantum numbers, for a precise mass measurement, and it may even allow a direct measurement of the Higgs-boson width. The CED production process with subsequent decay into bottom quarks is of particular relevance since this channel may be the only possibility for directly accessing the $h b \bar{b}$ coupling, although the decay into bottom quarks is by far the dominant decay mode of the lighter $\mathcal{C P}$-even Higgs boson in nearly the whole parameter space of the MSSM (and it is also the dominant decay of a light SM-like Higgs). For this reason information on the bottom Yukawa coupling is important for determining any Higgs-boson coupling at the LHC (rather than just ratios of couplings), see Ref. [13].

Figs. 3, 4, show that at the $3 \sigma$ level a significantly larger part of the $M_{A}-\tan \beta$ plane can be covered compared to the $5 \sigma$ discovery contours. In particular, in the "600 $\mathrm{fb}^{-1} \mathrm{eff} \times 2$ " scenario the coverage in both benchmark scenarios extends to large $M_{A}$ values and small values of $\tan \beta$. With the exception of a small parameter region around $M_{A} \approx 140(130) \mathrm{GeV}$, in the $M_{h}^{\max }$ (no-mixing) scenario the whole $M_{A}-\tan \beta$ plane of the MSSM (and also the case of a light SM-like Higgs) can be covered with the CED process in this case. This important result implies that if the CED channel can be utilised at high instantaneous luminosity (which requires in particular that pile-up background is brought under control, see the discussion in Sect. 5) there is a good chance to detect the lighter $\mathcal{C} \mathcal{P}$-even Higgs boson of the MSSM in this channel with subsequent decay into bottom quarks, yielding crucial information on the properties of the new state.

In Fig. 5 we show the results for $h \rightarrow \tau^{+} \tau^{-}$in the $M_{h}^{\max }$ scenario (with $\mu=+200 \mathrm{GeV}$ ). Despite the fact that this decay is easier to disentangle from the background than the $h \rightarrow b \bar{b}$ channel, the lower branching ratio compared to the decay $h \rightarrow b \bar{b}$ results in a slightly worse coverage of the MSSM parameter space in the $M_{A}-\tan \beta$ plane. While in the "60 $\mathrm{fb}^{-1 "}$ scenario the $b \bar{b}$ channel allows the region of low $M_{A}$ and high $\tan \beta$ to be probed at the $5 \sigma$ level (see Fig. 3), the statistical significance achievable with the $\tau^{+} \tau^{-}$channel at the same luminosity remains below $5 \sigma$ for $\tan \beta<50$. For the most optimistic luminosity scenarios the coverage of the $\tau^{+} \tau^{-}$channel is only slightly worse than that of the $b \bar{b}$ channel shown in Fig. 3. The qualitative features of the no-mixing scenario are similar to those discussed above for the $h \rightarrow b \bar{b}$ channel, and we do not show it here. In interpreting the prospects for the $h \rightarrow \tau^{+} \tau^{-}$channel it should be noted that we have conservatively assumed the same selection efficiencies for this channel as for the $h \rightarrow b \bar{b}$ channel. As discussed above, an improved selection procedure could yield a significant gain for the $\tau^{+} \tau^{-}$channel. 

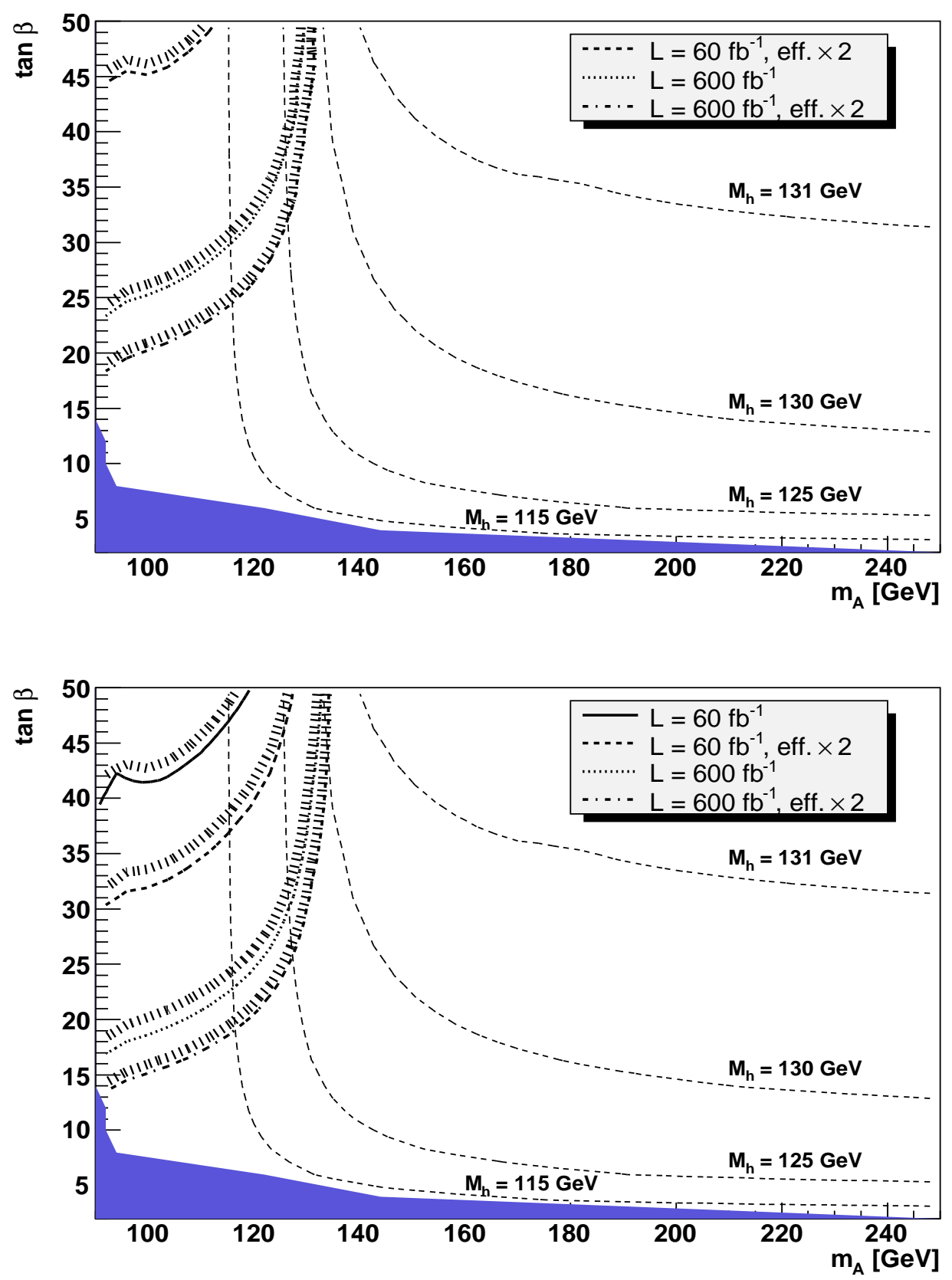

Figure 5: $5 \sigma$ discovery contours (upper plot) and contours of $3 \sigma$ statistical significance (lower plot) for the $h \rightarrow \tau^{+} \tau^{-}$channel in CED production in the $M_{A}-\tan \beta$ plane of the MSSM within the $M_{h}^{\max }$ benchmark scenario. The results are shown for assumed effective luminosities (see text, combining ATLAS and CMS) of $60 \mathrm{fb}^{-1}, 60 \mathrm{fb}^{-1} \mathrm{eff} \times 2,600 \mathrm{fb}^{-1}$ and $600 \mathrm{fb}^{-1}$ eff $\times 2$. The values of the mass of the light $\mathcal{C P}$-even Higgs boson, $M_{h}$, are indicated by contour lines. The dark shaded (blue) region corresponds to the parameter region that is excluded by the LEP Higgs searches in the channel $e^{+} e^{-} \rightarrow Z^{*} \rightarrow Z h, H[51,52]$. 


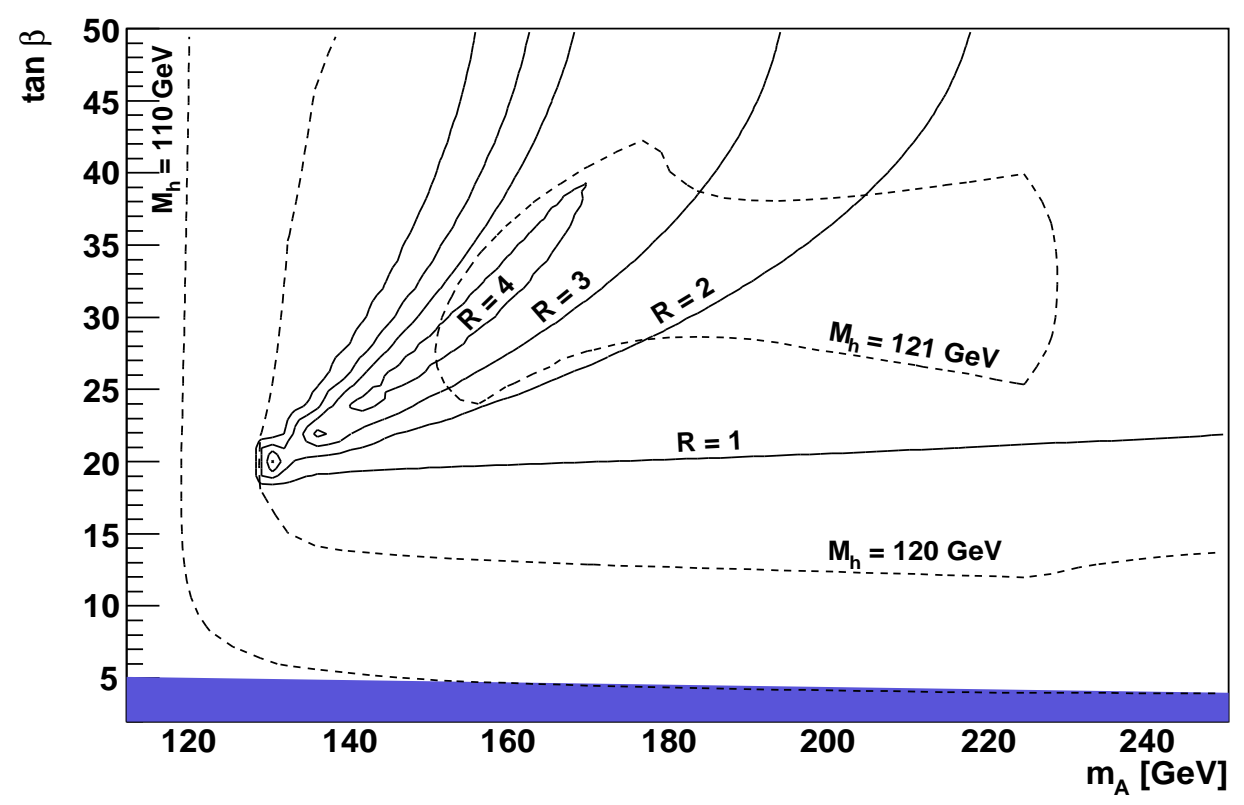

Figure 6: Contours for the ratio of MSSM to SM signal events in the $h \rightarrow W W^{(*)}$ channel in CED production in the $M_{A}-\tan \beta$ plane (with $M_{H^{\mathrm{Sm}}}=M_{h}$ ). The ratio is shown in the small- $\alpha_{\text {eff }}$ benchmark scenario. The values of the mass of the light $\mathcal{C} \mathcal{P}$-even Higgs boson, $M_{h}$, are indicated by dashed contour lines. The dark shaded (blue) region corresponds to the parameter region that is excluded by the LEP Higgs searches in the channel $e^{+} e^{-} \rightarrow Z^{*} \rightarrow$ $Z h, H[51,52]$.

As a further channel for the lighter $\mathcal{C} \mathcal{P}$-even Higgs boson of the MSSM we consider the decay $h \rightarrow W W^{(*)}$. Since the irreducible background to this channel has not yet been fully investigated, it would be premature to present $5 \sigma$ discovery regions as for the other channels. Thus we only present the ratio of signal events in the MSSM to those in the SM (with $M_{H^{\mathrm{SM}}}=M_{h}$ ). As discussed above, an enhancement of the $h b \bar{b}$ coupling compared to the SM case typically occurs in the region of large $\tan \beta$ and small $M_{A}$, see Fig. 2. This in turn leads to a reduction of the branching ratio of $h \rightarrow W W^{(*)}$, so that one would expect that in general the $h \rightarrow W W^{(*)}$ channel in the MSSM should have a smaller or at most equal event rate compared to the corresponding process in the SM (with $M_{H \mathrm{SM}}=M_{h}$ ). However, as discussed in Sect. 2 in the context of the small- $\alpha_{\text {eff }}$ benchmark scenario, higher-order corrections in the MSSM can also have the opposite effect and yield a significant suppression of the $h b \bar{b}$ coupling, giving rise to an enhancement of $\mathrm{BR}\left(h \rightarrow W W^{(*)}\right)$. Fig. 6] shows contours for the ratio of signal events in the MSSM to those in the SM in the $h \rightarrow W W^{(*)}$ channel within the $M_{A}-\tan \beta$ plane of the small- $\alpha_{\text {eff }}$ benchmark scenario, see eq. (14). For $140 \mathrm{GeV} \lesssim M_{A} \lesssim 170 \mathrm{GeV}$ and intermediate $\tan \beta$ an enhancement of the MSSM rate compared to the SM case of up to a factor of $R=4$ is possible. In this region one would expect about 12 signal events in CED Higgs production with $h \rightarrow W W^{(*)}$ for a luminosity of $60 \mathrm{fb}^{-1}$ within the acceptance of the Roman Pots at 420 and $220 \mathrm{~m}$, and after applying the standard lepton Level 1 trigger thresholds. 


\subsection{Prospective sensitivities for CED production of the heavy $\mathcal{C} \mathcal{P}$ - even Higgs boson}

We now turn to the prospects for producing the heavier $\mathcal{C} \mathcal{P}$-even Higgs boson of the MSSM in CED channels. The discovery reach in the "conventional" search channels at the LHC, in particular $b \bar{b} H / A, H / A \rightarrow \tau^{+} \tau^{-}$, covers the parameter region of high $\tan \beta$ and not too large $M_{A}[1-4]$, while a "wedge region" $[1,4,36]$ remains where the heavy MSSM Higgs bosons escape detection at the LHC (the discovery reach is somewhat extended if decays of the heavy MSSM Higgs bosons into supersymmetric particles can be utilised [1,4]). CED production of the heavier $\mathcal{C} \mathcal{P}$-even Higgs boson of the MSSM with subsequent decay into bottom quarks provides a unique opportunity for accessing its bottom Yukawa coupling in a mass range where for a SM Higgs boson the decay rate into bottom quarks would be negligibly small.

As explained above, the properties of the heavier $\mathcal{C} \mathcal{P}$-even Higgs boson of the MSSM differ very significantly from the ones of a SM Higgs in the region where $M_{H}, M_{A} \gtrsim 150 \mathrm{GeV}$. While for a SM Higgs the $\mathrm{BR}(H \rightarrow b \bar{b})$ is strongly suppressed in this mass region, the decay into bottom quarks is the dominant decay mode for the heavier $\mathcal{C} \mathcal{P}$-even MSSM Higgs boson (as long as no decays into supersymmetric particles or lighter Higgs bosons are open). The drastic difference between the heavier $\mathcal{C} \mathcal{P}$-even MSSM Higgs boson and its SM counterpart with the same mass is clearly visible in Figs. 7, 8, where the ratio of signal events for the MSSM over the events for the SM is displayed in the $M_{A}-\tan \beta$ plane for the $M_{h}^{\max }$ (upper plots) and nomixing (lower plots) benchmark scenarios with $\mu=+200 \mathrm{GeV}$ (Fig. 7) and $\mu=-500 \mathrm{GeV}$ (Fig. 8). For illustration, contour lines for the mass of the heavier $\mathcal{C} \mathcal{P}$-even Higgs boson are also given. As before, the dark shaded (blue) region indicates the part of the $M_{A}$-tan $\beta$ plane that is excluded by the LEP Higgs searches [52] in the channel $e^{+} e^{-} \rightarrow Z^{*} \rightarrow Z h, H[51,52]$.

Fig. 7 shows that the MSSM process in the $H \rightarrow b \bar{b}$ channel is significantly enhanced compared to the SM case essentially everywhere in the unexcluded part of the $M_{A}-\tan \beta$ plane. For $M_{A} \approx 120 \mathrm{GeV}$ the rate is enhanced by about a factor of $R=3$, while for large $M_{A}$ we find a huge enhancement by typically two orders of magnitude. In accordance with our discussion of the $\Delta_{b}$ corrections given above, the enhancement is somewhat more pronounced in the no-mixing scenario, where it reaches a factor of $R=500$ in the high-tan $\beta$ region, while Fig. 7 shows an enhancement of up to $R=400$ in the $M_{h}^{\max }$ scenario.

While in the case of positive $\mu$ shown in Fig. 7 the higher-order contribution $\Delta_{b}$ yields a suppression of the bottom Yukawa coupling, the opposite effect occurs if the parameter $\mu$ is negative. This is illustrated in Fig. 8, where $\mu=-500 \mathrm{GeV}$ has been chosen. As a consequence, the enhancement compared to the SM rate is even larger in this case, reaching a factor of $R=2000$ in the $M_{h}^{\max }$ scenario. (The fact that the enhancement in the no-mixing scenario with $\mu=-500 \mathrm{GeV}$ is less pronounced is due to the numerically smaller value of $\Delta_{b}$ in the no-mixing scenario, as explained above.)

In Figs. 911 we show the contours of $5 \sigma$ (upper plots) and $3 \sigma$ (lower plots) statistical significances obtained in the four luminosity scenarios specified above for the $H \rightarrow b \bar{b}$ channel in CED production within the $M_{h}^{\max }$ scenario for $\mu=+200 \mathrm{GeV}$, the no-mixing scenario for $\mu=+200 \mathrm{GeV}$ and the $M_{h}^{\max }$ scenario for $\mu=-500 \mathrm{GeV}$, respectively. The pattern of the $5 \sigma$ and $3 \sigma$ contours follows from the discussion above: the coverage in the $M_{A}-\tan \beta$ plane is largest in the $M_{h}^{\max }$ scenario for $\mu=-500 \mathrm{GeV}$ (Fig. 11), followed by the no-mixing scenario for $\mu=+200 \mathrm{GeV}$ (Fig. 10) and the $M_{h}^{\max }$ scenario for $\mu=+200 \mathrm{GeV}$ (Fig. 9). 

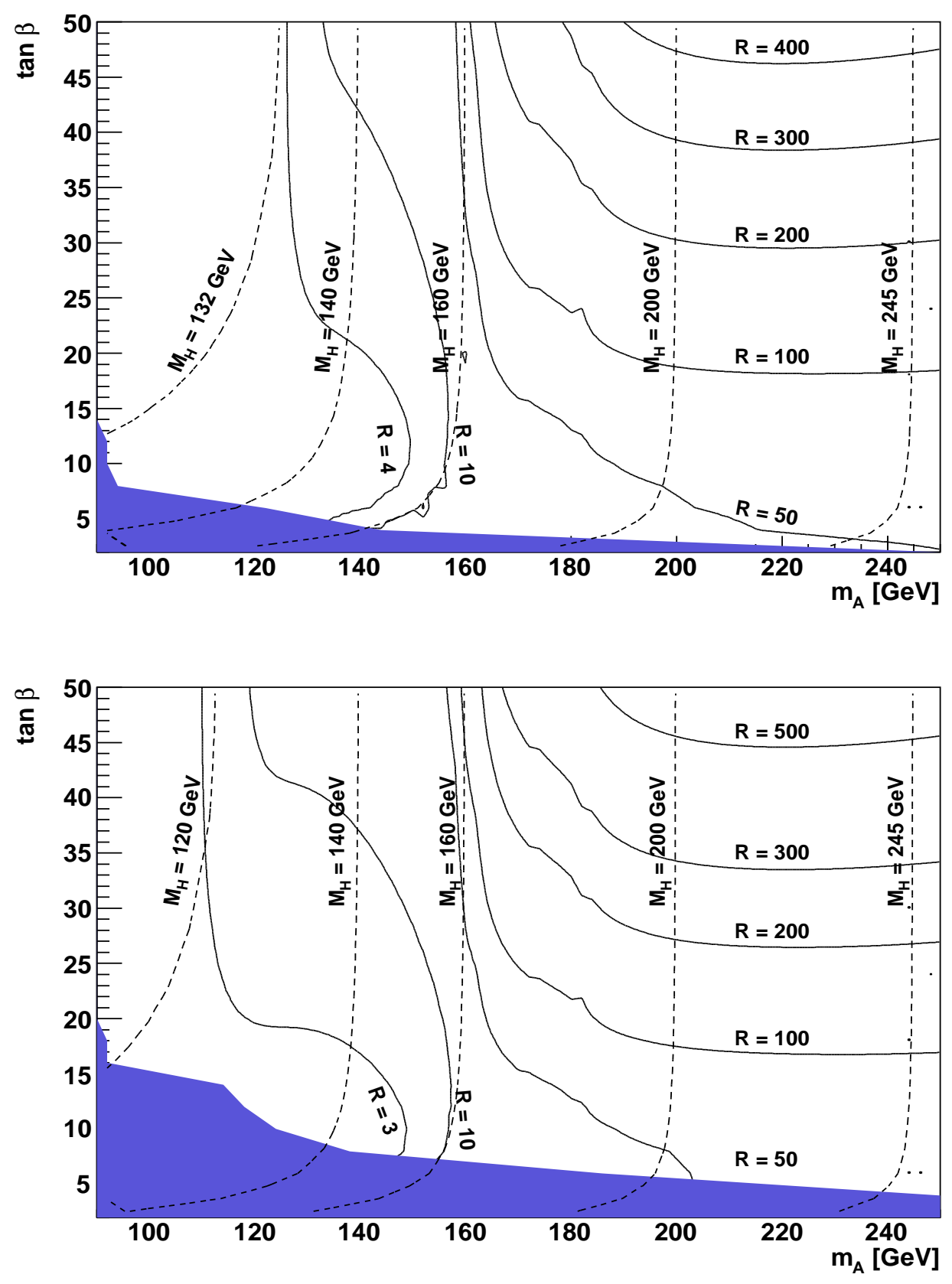

Figure 7: Contours for the ratio of signal events in the MSSM to those in the SM in the $H \rightarrow b \bar{b}$ channel in CED production in the $M_{A}-\tan \beta$ plane. The ratio is shown in the $M_{h}^{\max }$ benchmark scenario (with $\mu=+200 \mathrm{GeV}$, upper plot) and in the no-mixing scenario (with $\mu=+200 \mathrm{GeV}$, lower plot). The values of the mass of the heavier $\mathcal{C} \mathcal{P}$-even Higgs boson, $M_{H}$, are indicated by dashed contour lines. The dark shaded (blue) region corresponds to the parameter region that is excluded by the LEP Higgs searches in the channel $e^{+} e^{-} \rightarrow Z^{*} \rightarrow Z h, H[51,52]$. 

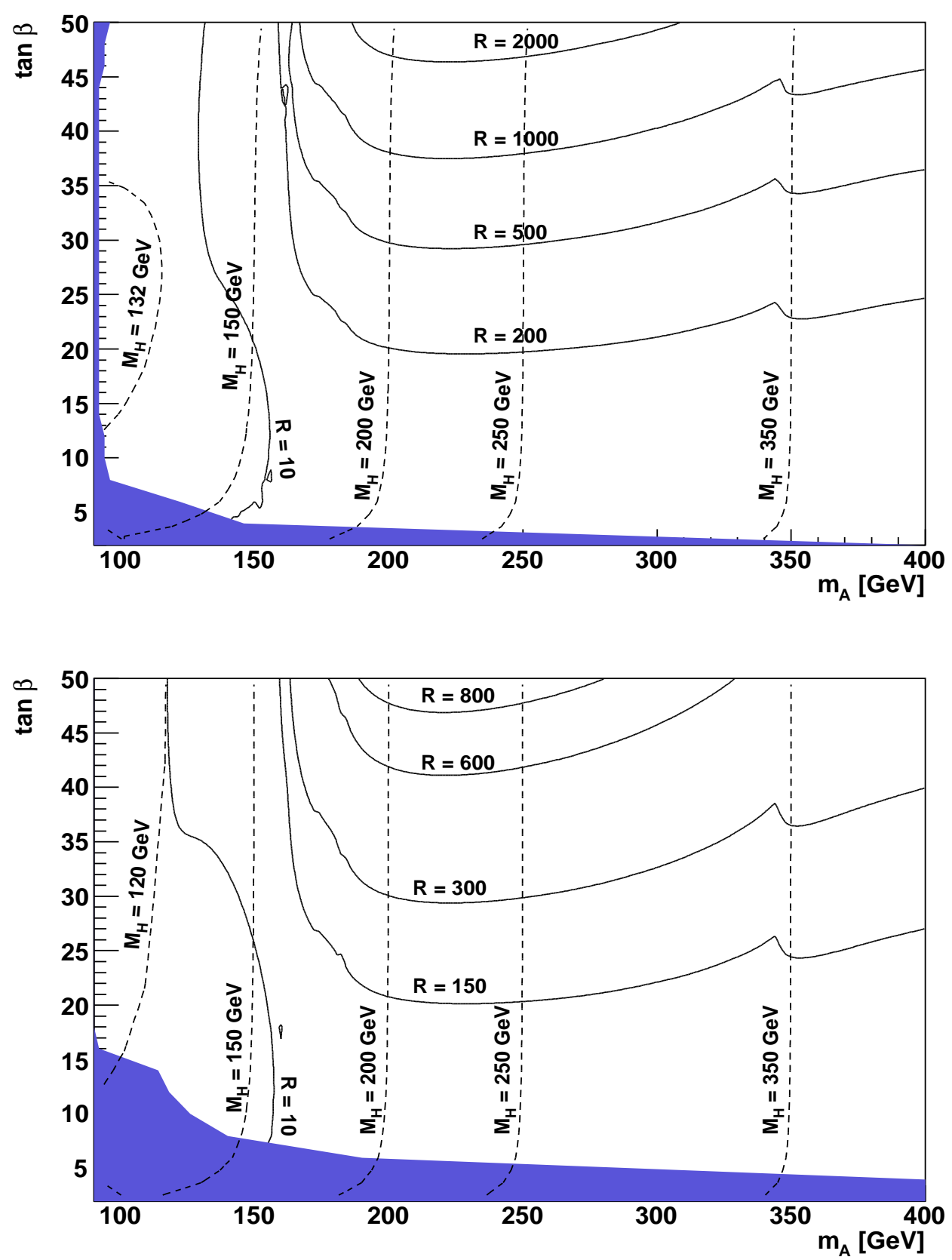

Figure 8: Contours for the ratio of signal events in the MSSM to those in the SM in the $H \rightarrow b \bar{b}$ channel in CED production in the $M_{A}-\tan \beta$ plane. The ratio is shown in the $M_{h}^{\max }$ benchmark scenario (with $\mu=-500 \mathrm{GeV}$, upper plot) and in the no-mixing scenario (with $\mu=-500 \mathrm{GeV}$, lower plot). The values of the mass of the heavier $\mathcal{C} \mathcal{P}$-even Higgs boson, $M_{H}$, are indicated by dashed contour lines. The dark shaded (blue) region corresponds to the parameter region that is excluded by the LEP Higgs searches in the channel $e^{+} e^{-} \rightarrow Z^{*} \rightarrow Z h, H[51,52]$. 

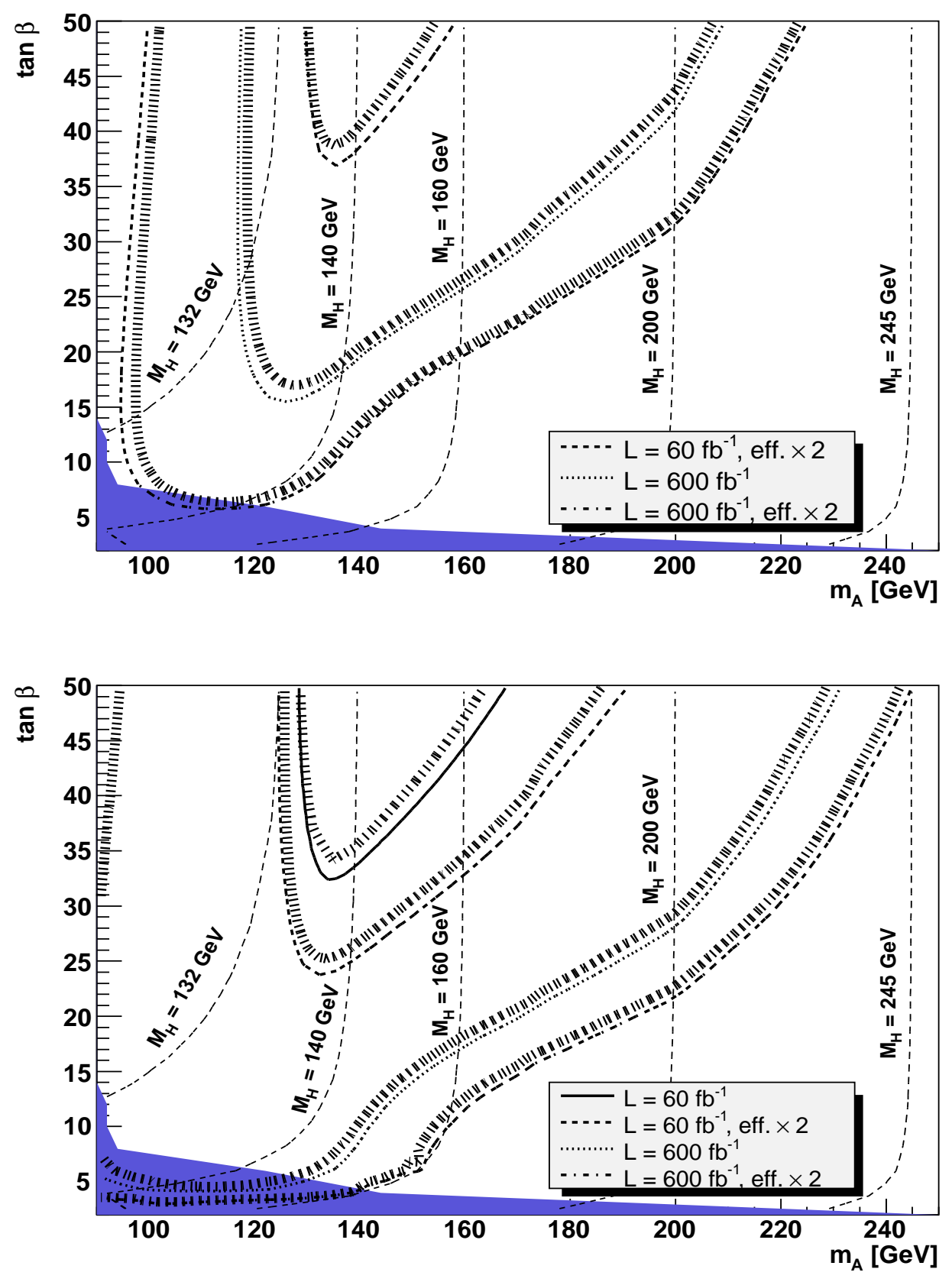

Figure 9: $5 \sigma$ discovery contours (upper plot) and contours of $3 \sigma$ statistical significance (lower plot) for the $H \rightarrow b \bar{b}$ channel in CED production in the $M_{A}-\tan \beta$ plane of the MSSM within the $M_{h}^{\max }$ benchmark scenario (with $\mu=+200 \mathrm{GeV}$ ). The results are shown for assumed effective luminosities (see text, combining ATLAS and CMS) of $60 \mathrm{fb}^{-1}, 60 \mathrm{fb}^{-1}$ eff $\times 2,600$ $\mathrm{fb}^{-1}$ and $600 \mathrm{fb}^{-1} \mathrm{eff} \times 2$. The values of the mass of the heavier $\mathcal{C} \mathcal{P}$-even Higgs boson, $M_{H}$, are indicated by contour lines. The dark shaded (blue) region corresponds to the parameter region that is excluded by the LEP Higgs searches in the channel $e^{+} e^{-} \rightarrow Z^{*} \rightarrow Z h, H[51,52]$. 

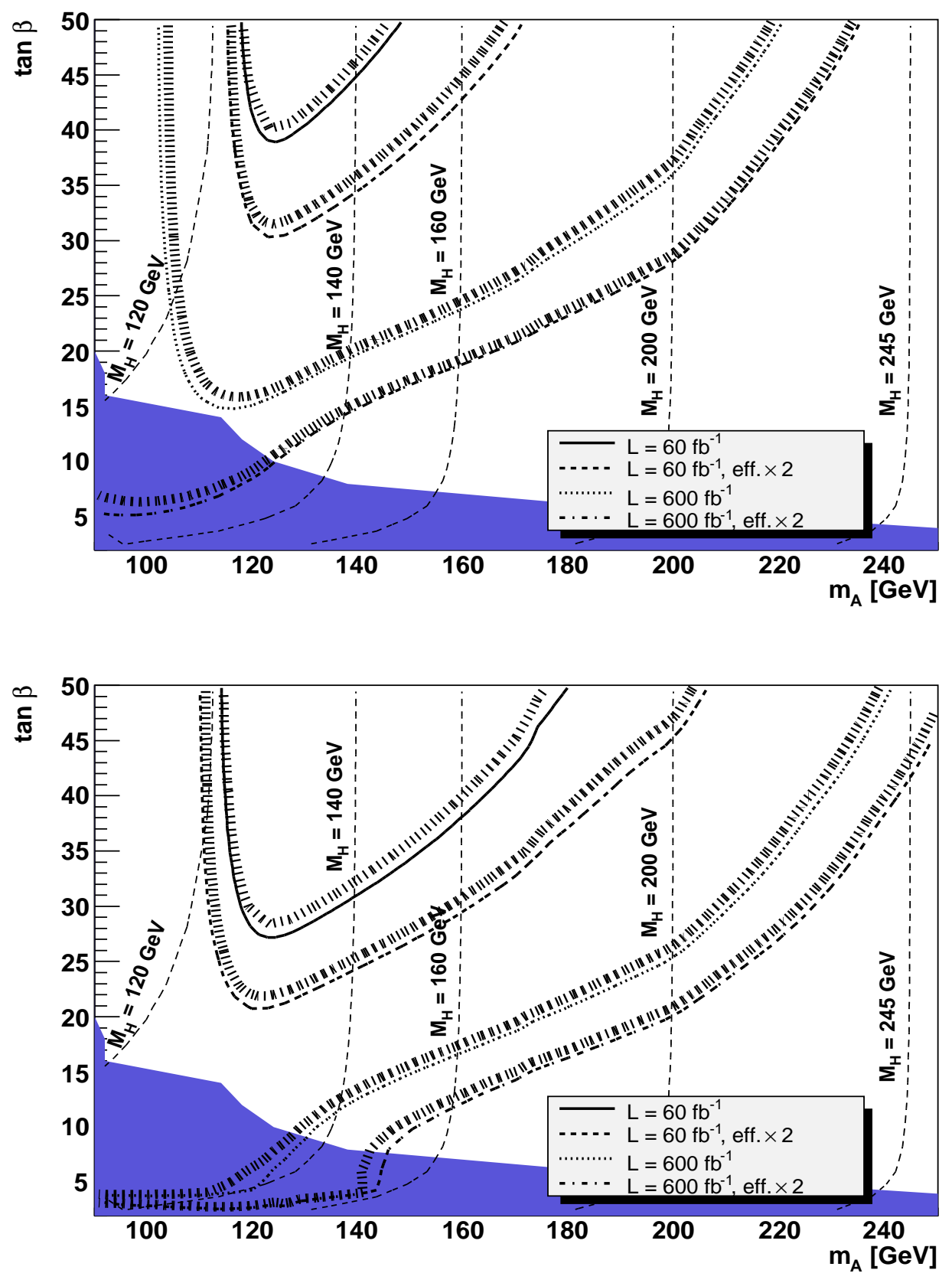

Figure 10: $5 \sigma$ discovery contours (upper plot) and contours of $3 \sigma$ statistical significance (lower plot) for the $H \rightarrow b \bar{b}$ channel in CED production in the $M_{A}-\tan \beta$ plane of the MSSM within the no-mixing benchmark scenario (with $\mu=+200 \mathrm{GeV}$ ). The results are shown for assumed effective luminosities (see text, combining ATLAS and CMS) of $60 \mathrm{fb}^{-1}, 60$ $\mathrm{fb}^{-1}$ eff $\times 2,600 \mathrm{fb}^{-1}$ and $600 \mathrm{fb}^{-1}$ eff $\times 2$. The values of the mass of the heavier $\mathcal{C} \mathcal{P}$-even Higgs boson, $M_{H}$, are indicated by contour lines. The dark shaded (blue) region corresponds to the parameter region that is excluded by the LEP Higgs searches in the channel $e^{+} e^{-} \rightarrow Z^{*} \rightarrow$ $Z h, H[51,52]$. 

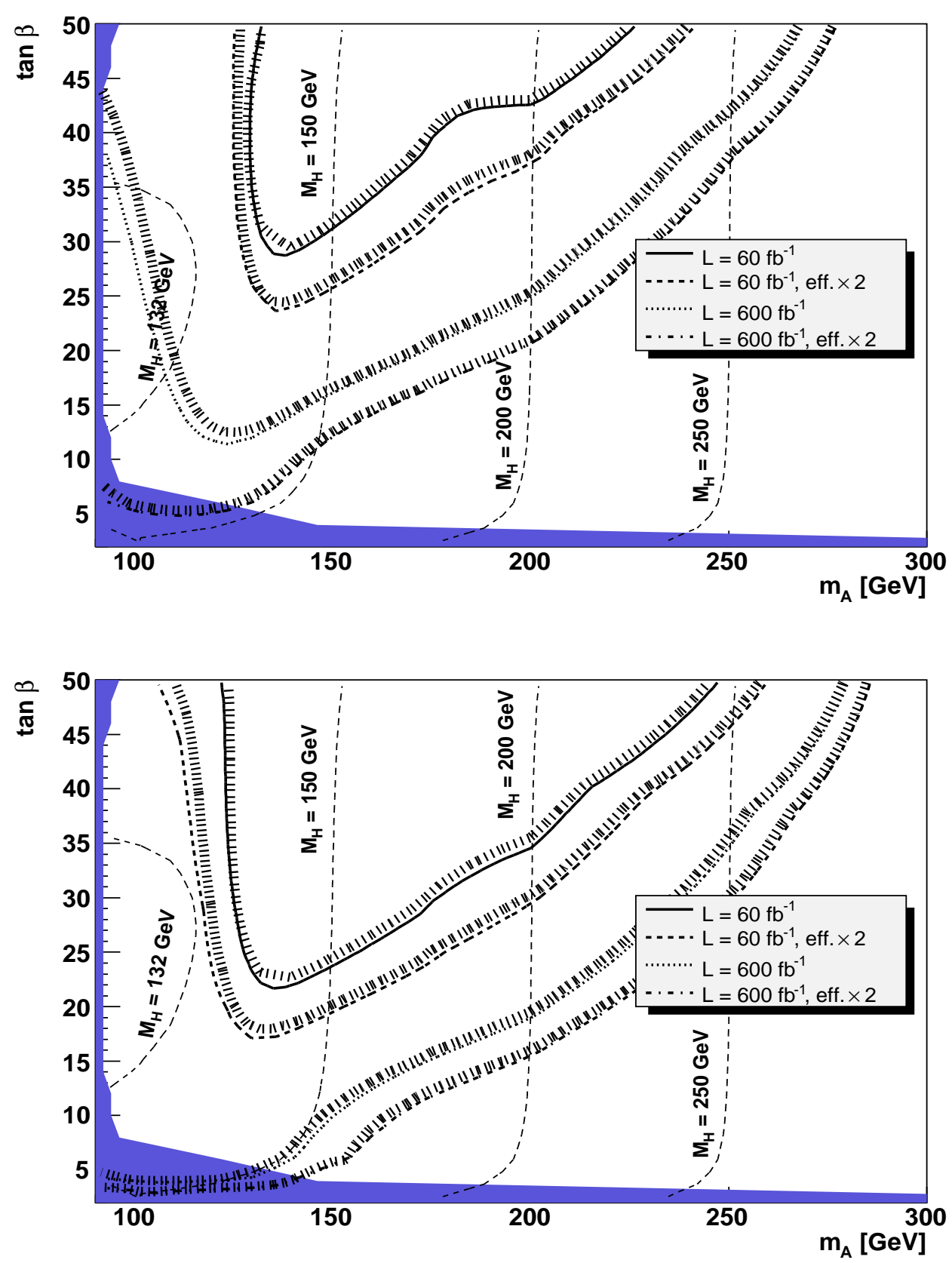

Figure 11: $5 \sigma$ discovery contours (upper plot) and contours of $3 \sigma$ statistical significance (lower plot) for the $H \rightarrow b \bar{b}$ channel in CED production in the $M_{A}$-tan $\beta$ plane of the MSSM within the $M_{h}^{\max }$ benchmark scenario for the case where $\mu=-500 \mathrm{GeV}$. The results are shown for assumed effective luminosities (see text, combining ATLAS and CMS) of 60 $\mathrm{fb}^{-1}, 60 \mathrm{fb}^{-1} \mathrm{eff} \times 2,600 \mathrm{fb}^{-1}$ and $600 \mathrm{fb}^{-1} \mathrm{eff} \times 2$. The values of the mass of the heavier $\mathcal{C P}$-even Higgs boson, $M_{H}$, are indicated by contour lines. The dark shaded (blue) region corresponds to the parameter region that is excluded by the LEP Higgs searches in the channel $e^{+} e^{-} \rightarrow Z^{*} \rightarrow Z h, H[51,52]$. 
In the $M_{h}^{\max }$ scenario for $\mu=+200 \mathrm{GeV}$ (Fig. 9) an integrated luminosity of $60 \mathrm{fb}^{-1}$ will not be sufficient to probe a parameter region with $\tan \beta \leq 50$ at the $5 \sigma$ level. At the $3 \sigma$ level, on the other hand, the sensitivity of the "60 $\mathrm{fb}^{-1}$ " scenario extends down to about $\tan \beta=35$ for $M_{A} \approx 130 \mathrm{GeV}$. In the " $600 \mathrm{fb}^{-1}$ eff $\times 2$ " scenario the discovery reach for the heavier $\mathcal{C} \mathcal{P}$-even Higgs boson goes beyond $M_{H} \approx 200 \mathrm{GeV}$ in the large $\tan \beta$ region at the $5 \sigma$ level, while at the $3 \sigma$ level the coverage extends to about $M_{H}=250 \mathrm{GeV}$ for $\tan \beta \approx 50$. In the "600 $\mathrm{fb}^{-1} \mathrm{eff} \times 2$ " scenario the $(5 \sigma$ level) discovery of a heavy $\mathcal{C} \mathcal{P}$-even Higgs boson with a mass of about $140 \mathrm{GeV}$ will be possible for all values of $\tan \beta$. This is of particular interest in view of the "wedge region" left uncovered by the conventional search channels for heavy MSSM Higgs bosons.

The search reach is somewhat extended in the no-mixing scenario for $\mu=+200 \mathrm{GeV}$ (Fig. 10). In this case the " $60 \mathrm{fb}^{-1}$ " scenario can probe $\tan \beta$ values down to about $\tan \beta=40$ for $M_{A} \approx 130 \mathrm{GeV}$ at the $5 \sigma$ level, while the coverage extends to $\tan \beta \gtrsim 30$ at the $3 \sigma$ level for small $M_{A}$. As an example of anticipated event rates, for $60 \mathrm{fb}^{-1}$ one would expect after all cuts in the no-mixing scenario for $\mu=+200 \mathrm{GeV}, M_{H} \approx 125 \mathrm{GeV}$ and $\tan \beta=50$ $(\tan \beta=40)$ about 43 signal events and 26 background events (28 signal events and 21 background events).

The coverage is further enhanced in the $M_{h}^{\max }$ scenario for $\mu=-500 \mathrm{GeV}$ (Fig. 11). In this case a significant part of the $M_{A}-\tan \beta$ plane can be probed with $60 \mathrm{fb}^{-1}$ at the $5 \sigma$ level, extending down to about $\tan \beta=30$ for $M_{A} \approx 140 \mathrm{GeV}$. At the $3 \sigma$ level the reach of the "60 $\mathrm{fb}^{-1 "}$ "scenario extends almost down to $\tan \beta=20$ for $M_{A} \approx 140 \mathrm{GeV}$. In this scenario the expected event rates for $60 \mathrm{fb}^{-1}$ are about 124 signal events and 65 background events (25 signal events and 14 background events) for $M_{H} \approx 140 \mathrm{GeV}$ and $\tan \beta=50$ $(\tan \beta=30)$. In the "600 $\mathrm{fb}^{-1}$ eff $\times 2 "$ scenario a heavy $\mathcal{C} \mathcal{P}$-even Higgs boson with a mass of almost $M_{H}=150 \mathrm{GeV}$ can be discovered at the $5 \sigma$ level for all values of $\tan \beta$. In the high $\tan \beta$ region, for this luminosity masses of $M_{H} \approx M_{A}$ in excess of $250 \mathrm{GeV}$ can be probed at the $5 \sigma$ level (the coverage only slightly increases at the $3 \sigma$ level). This means that CED production at the LHC may be a unique way to access the bottom Yukawa coupling of a Higgs boson as heavy as $250 \mathrm{GeV}$ (which would obviously be a clear sign of physics beyond the Standard Model).

The heavier $\mathcal{C P}$-even Higgs boson can also be detected via the decay $H \rightarrow \tau^{+} \tau^{-}$. The dependence on the parameter $\mu$ is less pronounced in this channel compared to CED production with the decay $H \rightarrow b \bar{b}$. This is due to the fact that the $\Delta_{b}$ corrections largely compensate between the production and the decay process, see Refs. [36,50] for a discussion of the analogous effect in the $b \bar{b} H / A, H / A \rightarrow \tau^{+} \tau^{-}$channel. We therefore restrict our discussion to the $M_{h}^{\max }$ scenario with $\mu=+200 \mathrm{GeV}$, see Fig. 12, The upper plot shows the $5 \sigma$ discovery contours, while the lower plot shows the contours of $3 \sigma$ statistical significance. Due to the suppressed branching ratio the discovery region is significantly smaller than for the decay $H \rightarrow b \bar{b}$ (although the enhancement of the signal rate compared to the SM case (not shown) is of similar size as for the $H \rightarrow b \bar{b}$ channel). At the $5 \sigma$ level an integrated luminosity of $600 \mathrm{fb}^{-1}$ is necessary to probe parameter regions with $\tan \beta \leq 50$. In the "600 $\mathrm{fb}^{-1} \mathrm{eff} \times 2$ " scenario the coverage extends to $M_{H} \approx 200 \mathrm{GeV}$ at the $3 \sigma$ level for $\tan \beta \approx 50$. 

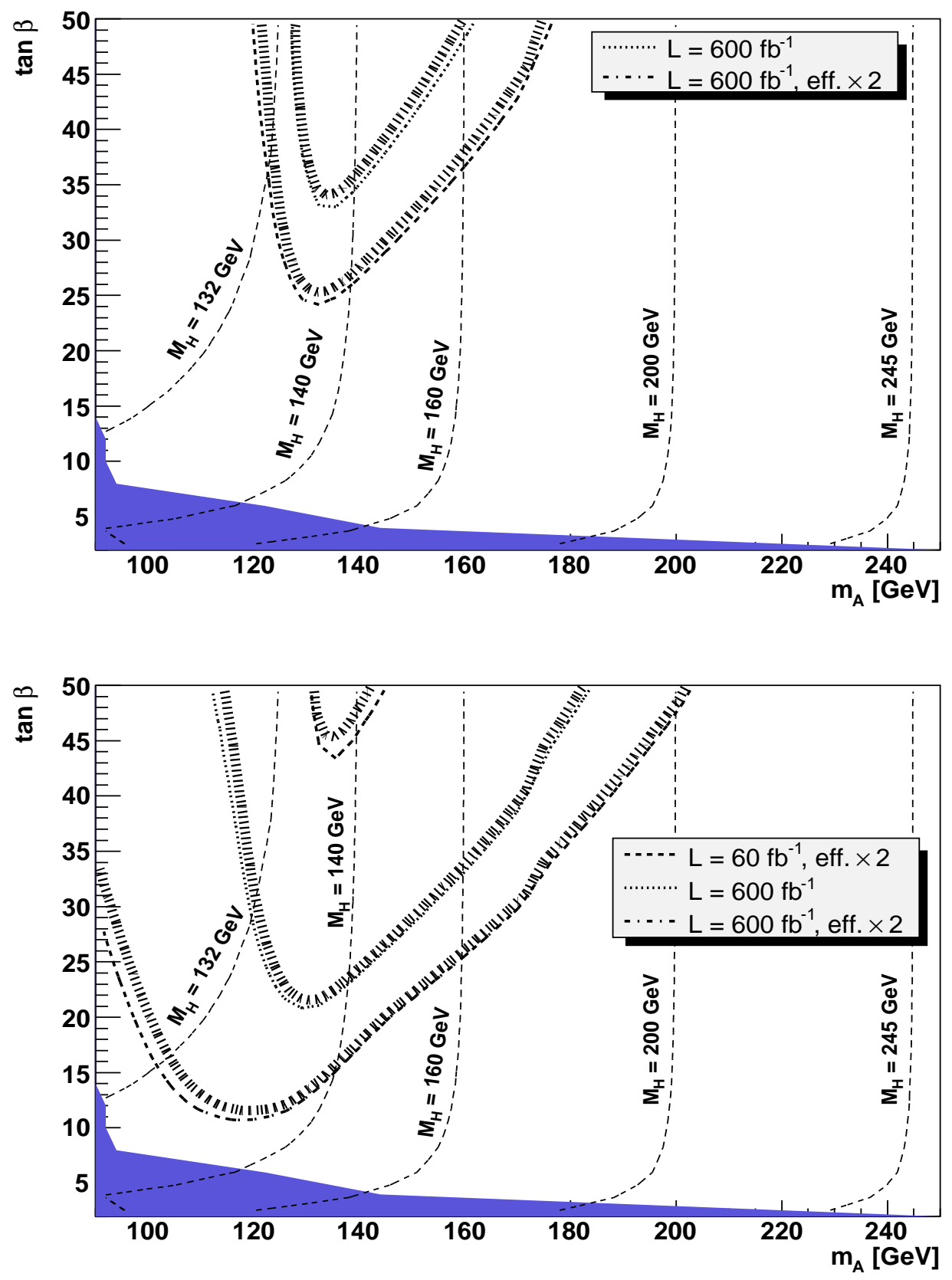

Figure 12: $5 \sigma$ discovery contours (upper plot) and contours of $3 \sigma$ statistical significance (lower plot) for the $H \rightarrow \tau^{+} \tau^{-}$channel in CED production in the $M_{A}-\tan \beta$ plane of the MSSM within the $M_{h}^{\max }$ benchmark scenario (with $\mu=+200 \mathrm{GeV}$ ). The results are shown for assumed effective luminosities (see text, combining ATLAS and CMS) of $60 \mathrm{fb}^{-1}, 60$ $\mathrm{fb}^{-1} \mathrm{eff} \times 2,600 \mathrm{fb}^{-1}$ and $600 \mathrm{fb}^{-1} \mathrm{eff} \times 2$. The values of the mass of the heavier $\mathcal{C} \mathcal{P}$-even Higgs boson, $M_{H}$, are indicated by contour lines. The dark shaded (blue) region corresponds to the parameter region that is excluded by the LEP Higgs searches in the channel $e^{+} e^{-} \rightarrow Z^{*} \rightarrow$ $Z h, H[51,52]$. 


\section{Pseudoscalar Higgs-boson production in diffractive processes}

As is well known (see for example Refs. [1-4]), identifying the pseudoscalar Higgs boson at the LHC will be a challenging task. In particular, in the case of the MSSM (and many other theories with extended Higgs sectors) the $\mathcal{C P}$-odd $A$ boson decouples from the vector bosons, and therefore neither Higgs-boson production via weak-boson fusion nor Higgs decay modes into gauge bosons are of practical use. Accordingly, it is of interest to investigate whether the diffractive mechanism can provide some additional leverage in the search for pseudoscalar Higgs bosons at the LHC.

Unfortunately, according to first studies performed in Ref. [33], the situation with diffractive mechanisms for $A$ boson-production in the MSSM looks less favourable compared to the case of CED production of $\mathcal{C} \mathcal{P}$-even Higgs bosons. Detailed future studies (both experimental and theoretical) are required in order to reach a firm conclusion regarding the prospects for detecting $A$ bosons in diffractive processes. The discussion below reflects our current (rather incomplete) understanding.

The main problem with the diffractive production mechanism for a heavy $0^{-}$state is the strong suppression (by about two orders of magnitude) of the CED mode as compared to the $0^{+}$case due to the P-even selection rule, see Sect. 3. Furthermore, for $M_{A} \gg M_{Z}$ the masses of the $\mathcal{C P}$-odd $A$ and the $\mathcal{C P}$-even $H$ are close to each other. Consequently, the $H$ contribution in CED production will completely dominate over the much smaller $A$ signal.

In order to evade the selection rule and to have a sizable and comparable $A$ and $H$ production rate, it was suggested in Ref. [33] to consider a less exclusive reaction

$$
p p \rightarrow X \oplus \phi \oplus Y \quad(\phi=A, H),
$$

where both incoming protons are allowed to dissociate, and the Higgs bosons $\phi=A, H$ are separated from the proton remnants by large rapidity gaps, see Fig. 13, As shown in Refs. $[15,16]$ such a 'semi-exclusive' process has the advantage of a much larger cross section than the CED case. For example, for Higgs-boson masses of 120-140 GeV, by requiring rapidity gaps $\Delta \eta>3$, the effective $g g^{P P}$-luminosity is enhanced by an order of magnitude. Moreover, the process in eq. (29) can be a good testing ground for the searches for a $\mathcal{C P}$ violating Higgs boson, see Ref. [35].

Despite its larger cross section, this semi-exclusive Higgs-boson production is much less advantageous for the $\mathcal{C P}$-even Higgs bosons as compared to the exclusive production analysed in Sect. 6. The main reason is that one is losing the selection rule which for the CED case gave rise to a strong suppression of the QCD backgrounds. We consider here semiexclusive production of the $\mathcal{C P}$-even Higgs bosons only as a background to the semi-exclusive $A$ searches, which seems to be the only prospective diffraction production channel for this process.

From the experimental perspective, observation of the $A$-boson production according to eq. (29) with large rapidity regions devoid of hadron radiation appears to be very challenging. In particular, an observation of a sizable signal rate would require a high LHC luminosity, $\mathcal{L}>10^{33} \mathrm{~cm}^{-2} \mathrm{sec}^{-1}$, where one faces experimental difficulties caused by the effects of pileup background. As discussed in Sect. [5, it might be possible to reduce this background 


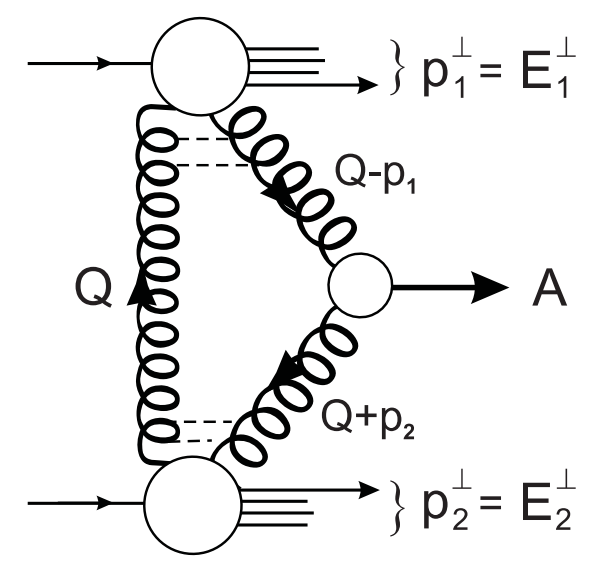

Figure 13: Central production of an $A$ boson with double diffractive dissociation ('semiexclusive' process), in which the incoming protons dissociate into systems with transverse momenta $p_{i}^{\perp}=E_{i}^{\perp}(i=1,2)$. $Q$ is the momentum of the exchanged gluon, $p_{1,2}$ are the momenta of the incoming particles.

to an acceptable level with the help of fast timing detectors and other techniques. The Level 1 trigger can be based, for example, on an observation in the forward calorimeters, at $\left|\eta_{1,2}\right| \sim 4-6$, of two unbalanced jets (i.e. one jet in the system $X$ and one in $Y$ ) with $E_{1,2}^{\perp}>20 \mathrm{GeV}$. The events with rapidity gaps can be selected subsequently in the off-line analysis. However, no dedicated experimental study of such a scenario has been performed so far. Let us recall that the signature for the Higgs production accompanied by two forward jets is a typical characteristics of the weak-boson fusion (WBF) mechanism [1,4]. There the standard procedure to reduce the QCD backgrounds is to use a (mini)jet veto. In the case of the semi-exclusive process of eq. (29) the characteristic transverse momenta of the forward jets are lower than in the WBF case (where they are typically around $10-20 \mathrm{GeV}$ ). Moreover, in contrast to WBF Higgs production the gaps are defined as rapidity regions devoid of any observed hadronic activity. This definition has often been used by the HERA experiments and is similar to that used by the CDF collaboration [66], especially in the case of the search for exclusive diphoton events [88]. Such a stronger veto on hadron activity in the rapidity gap interval should lead to a more pronounced angular correlation between the forward jets.

Since the main decay mode of the $A$ boson is into bottom quarks, with $\operatorname{BR}(A \rightarrow$ $b \bar{b}) \approx 90 \%$, the QCD background will be a serious obstacle for the semi-exclusive search for $A$ bosons. This is due in particular to the fact that in the semi-exclusive kinematics of the process in eq. (29) the $J_{z}=0$ selection rule can no longer be applied to suppress the $b \bar{b}$ background. Furthermore, the possibility of a very good missing-mass resolution, which was provided by the forward proton taggers in the exclusive reaction, does not of course exist for the semi-exclusive process.

In order to evaluate the expected signal and background cross sections, we use below a simplified parametrisation for the 'semi-exclusive' $g g^{P P}$ luminosity for production of a system of mass $M$, which was calculated at leading order in Ref. [16]. In the region of interest, $100 \lesssim M_{A} \lesssim 300 \mathrm{GeV}$, the semi-exclusive $g g^{P P}$ luminosity at $\Delta \eta>3$ and $E^{\perp}>20 \mathrm{GeV}$ can 
be approximated by

$$
\frac{M^{2} d \mathcal{L}_{\text {incl }}}{d M^{2}}=0.0024 \exp \left(-0.416\left(\frac{M}{100 \mathrm{GeV}}-2\right)\right) .
$$

The corresponding 'semi-exclusive' $A$-signal cross section is [16]

$$
\hat{\sigma}^{\text {incl }}=\frac{\pi^{2} \Gamma(A \rightarrow g g)}{M_{A}^{3}} \delta\left(1-M^{2} / M_{A}^{2}\right) .
$$

Thus, to compute the cross section $\sigma$ for the semi-exclusive production of the pseudoscalar $A$ the following formula

$$
\sigma \cdot \mathrm{BR}=0.0024 \exp \left(-0.416\left(\frac{M_{A}}{100 \mathrm{GeV}}-2\right)\right) \frac{\pi^{2} \Gamma(A \rightarrow g g)}{M_{A}^{3}} \mathrm{BR} \cdot 0.39 \mathrm{mb} \mathrm{GeV}^{2}
$$

can be used. Here BR is the corresponding branching fraction for $A \rightarrow b \bar{b}$ or $A \rightarrow \tau^{+} \tau^{-}$(as calculated with FeynHiggs [37-40]).

To evaluate the $b \bar{b}$ QCD background, we simply convolute the effective luminosity $\mathcal{L}_{\text {incl }}$ with the spin-summed leading-order cross section for the hard subprocess $g g \rightarrow b \bar{b}$, given in Ref. [16] (see also Ref. [89]). Thus, the signal-to-background ratio in the $b \bar{b}$ channel can be expressed as

$$
S / B \simeq \frac{\Gamma(A \rightarrow g g) \cdot \mathrm{BR}(A \rightarrow b \bar{b})}{0.25 \alpha_{s}^{2} \Delta M_{b b}} .
$$

Here $\Delta M_{b b}$ denotes, as before, the $b \bar{b}$ mass window. To illustrate the typical expectation for the signal-to-background ratio in the $A \rightarrow b \bar{b}$ channel we first consider a parameter point in the $M_{h}^{\max }$ scenario with $\mu= \pm 200 \mathrm{GeV}$ at $\tan \beta=30$ and $M_{A}=140 \mathrm{GeV}$. Unfortunately, the result for the signal-to-background ratio appears to be not encouraging. For a mass window $\Delta M_{b b} \simeq 24 \mathrm{GeV}$ (taken as twice the mass resolution in the central detector) and the angular cut $60^{\circ}<\theta<120^{\circ}$ to allow for the suppression of the collinear singularity in the background $g g \rightarrow b \bar{b}$ subprocess, we arrive at $S / B \sim 1 / 100$, with the signal being at the $\mathrm{fb}$ level. To gain insight into what one might expect at best in the $b \bar{b}$ channel, we focus on a 'most optimistic' $M_{h}^{\max }$ scenario with $\mu=-700 \mathrm{GeV}, \tan \beta=50$ and $m_{\tilde{g}}=1000 \mathrm{GeV}$. As discussed in Sect. 2.2, a large negative value of $\mu$ (together with large $\tan \beta$ and $m_{\tilde{g}}$ ) leads to a significant enhancement of the bottom Yukawa coupling of the $A$ boson. For this 'most optimistic' scenario we find at $M_{A}=160-200 \mathrm{GeV}$

$$
\Gamma(A \rightarrow g g) \cdot \operatorname{BR}(A \rightarrow b \bar{b}) \approx 22-24 \mathrm{MeV}
$$

and $\sigma \cdot \mathrm{BR}(A \rightarrow b b)$ is decreasing from $65 \mathrm{fb}$ for $M_{A}=160 \mathrm{GeV}$ to $25 \mathrm{fb}$ for $M_{A}=200 \mathrm{GeV}$.

This is illustrated in Fig. 14, where we show for the $M_{h}^{\max }$ scenario with $\mu=-700 \mathrm{GeV}$ and $m_{\tilde{g}}=1000 \mathrm{GeV}$ the production cross section for the $A$ boson (upper plot) and the ratio of signal events in the MSSM to those in the SM with $M_{H^{\mathrm{SM}}}=M_{A}$ (lower plot). The difference in the region exluded by the LEP Higgs searches in the channel $e^{+} e^{-} \rightarrow Z^{*} \rightarrow Z h, H[51,52]$ compared to the other plots in this paper results from a downward shift in $M_{h}$ caused by the enhanced bottom Yukawa coupling in the region of large $\tan \beta$. It should be noted that the 
large-tan $\beta$ region for $\mu=-700 \mathrm{GeV}$ is also affected by the limits from the MSSM Higgsboson searches at the Tevatron, in particular in the $b \bar{b}+(H, A), H, A \rightarrow b \bar{b}$ channel [53]. The cross section in Fig. 14 ranges from $1 \mathrm{fb}$ to $100 \mathrm{fb}$ for large $\tan \beta$. The ratio of $A$ signal events in the MSSM to those in the SM case (with $M_{H^{\mathrm{SM}}}=M_{A}$ ) reaches up to $R=100$ for $\tan \beta \lesssim 15$ or $M_{A} \lesssim 150 \mathrm{GeV}$. For large $\tan \beta$ and $M_{A}$ a ratio of up to $R=5000$ is possible. Accordingly, Fig. 14 gives an idea in which part of the MSSM parameter space there may be a chance for a successful $A$ search in the semi-exclusive process. In the discussed 'most optimistic' case, the signal-to-background ratio in the $b \bar{b}$ channel, using eq. (33), gives $S / B \approx 5.5 \mathrm{GeV} / \Delta M_{b b}$. Therefore, since the production cross section and branching ratio are already chosen at optimistic values, an immediate conclusion is that the prospects for hunting a $0^{-}$Higgs boson depend strongly on progress in improving $\Delta M_{b b}$.

Even with such an overwhelming QCD background there may still be a chance to identify the $A \rightarrow b \bar{b}$ signal by studying the azimuthal angular distributions of the transverse energy flows in the forward (and backward) regions of proton dissociation, i.e. of the total transverse momenta of the $X$ and $Y$ systems, see Fig. ${ }^{13}{ }^{16}$ The dependence on the azimuthal angle $\varphi$ between the total transverse momenta of the $X$ and $Y$ systems has the form

$$
\begin{aligned}
& \cos ^{2} \varphi \quad \text { for } H\left(0^{+}\right) \\
& \text {and } \sin ^{2} \varphi \text { for } A\left(0^{-}\right) \text {, }
\end{aligned}
$$

whereas the $\varphi$-dependence of the backgrounds is practically flat. The different behaviour of the azimuthal angular distributions of signal and background may therefore allow for an improvement in the statistical significance of the signal.

The difference in the azimuthal angular dependence may even provide a way to discriminate between $0^{+}$and $0^{-}$Higgs-boson production [33]. By selecting events with rather large $E_{1,2}^{\perp} \gtrsim 10-20 \mathrm{GeV}$, where the transverse energy flows in the forward and backward hemispheres are orthogonal to each other, the $H$ signal can be suppressed. In the region of $E_{1,2}^{\perp}>20 \mathrm{GeV}$ and $\left|\eta_{1,2}\right|=3-6$, for example, the T2 detector of TOTEM [25] and/or forward hadronic calorimeters (HF (CMS) or FCAL (ATLAS) and ZDC (CMS and ATLAS)) could be used for such a discrimination between $A$ and $H$. However, at the moment it is still unclear whether this is in fact a viable option. Before drawing a firm conclusion, the whole issue of experimental studies related to proton dissociation needs further detailed investigation.

We also briefly investigate diffractive $A$ production with subsequent decay to $\tau^{+} \tau^{-}$. Here the dominant non-pile-up backgrounds, arising from the QED production process $p p \rightarrow$ $X+\left(\tau^{+} \tau^{-}\right)+Y$ (see Ref. [35]) and from misidentification of a gluon dijet as a $\tau^{+} \tau^{-}$ system, are small, while the branching fraction $\operatorname{BR}\left(A \rightarrow \tau^{+} \tau^{-}\right)$is about $10 \%$. Using the cut $E_{\mathrm{T}}>20 \mathrm{GeV}$ the cross section for the $A \rightarrow \tau^{+} \tau^{-}$channel in the mass range $100-150 \mathrm{GeV}$ and $\tan \beta>20$ ranges between 1-2 fb. If the overall efficiency were about $4-10 \%$ as in the case of the $\tau^{+} \tau^{-}$channel in the exclusive processes (see Tab. 1), an integrated LHC luminosity of $\mathcal{L}=600 \mathrm{fb}^{-1}$ could be sufficient for the observation of the semi-exclusive Higgs-boson signal. Moreover, in such a case one could be even able to discriminate between the $A$ and $H$ bosons by studying the azimuthal angular distribution. However, this simple estimate may turn out to be too optimistic since various experimental issues affecting the semi-exclusive process at

\footnotetext{
${ }^{16}$ It should be noted that at $E^{\perp}>3-5 \mathrm{GeV}$ the energy flow is dominated by one (gluon) jet with the lowest rapidity.
} 

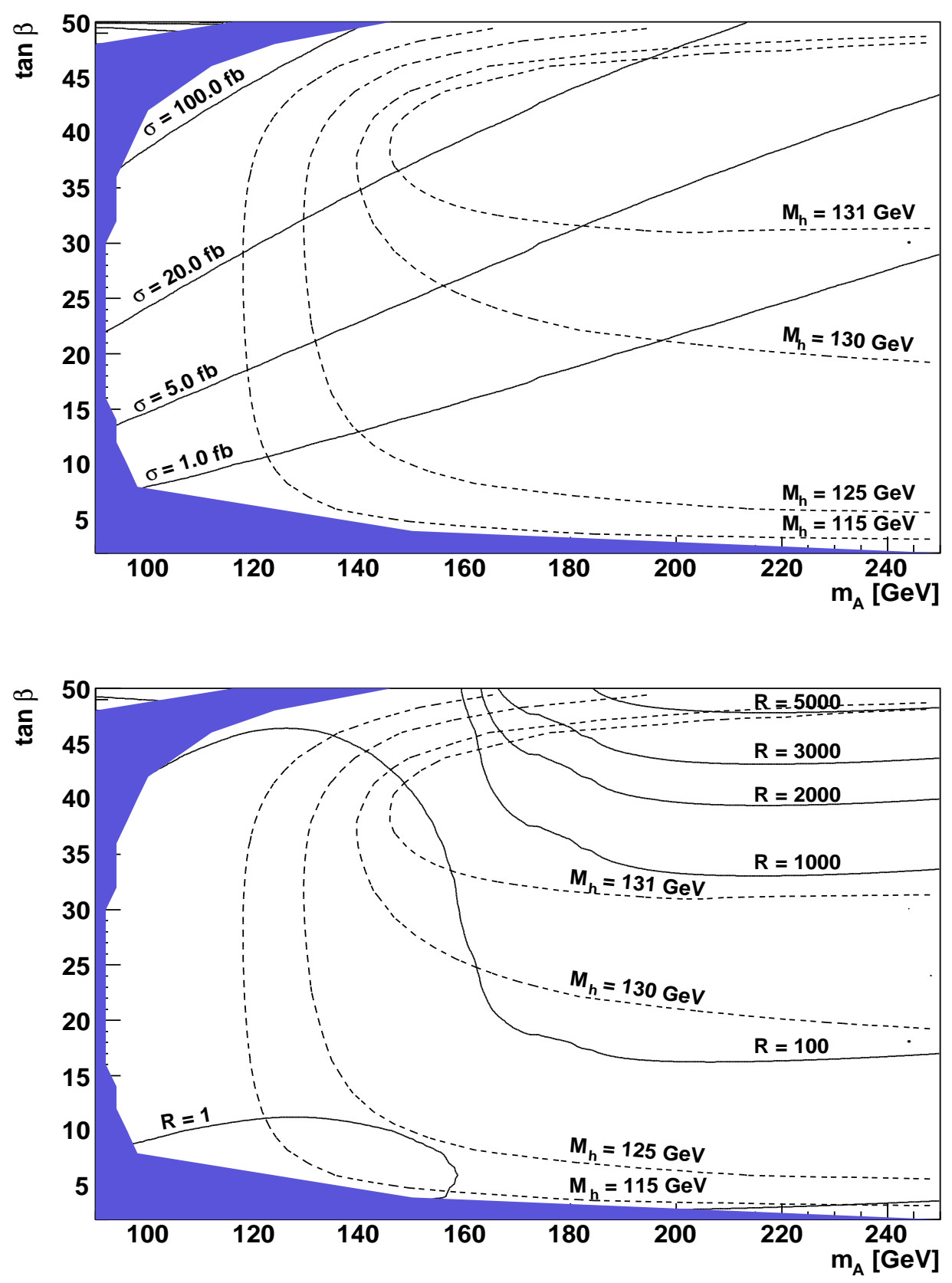

Figure 14: Upper plot: The cross section (in fb) for central production of the $A$ boson with double diffractive dissociation ('semi-exclusive' process) in the $M_{A}$-tan $\beta$ plane for the $M_{h}^{\max }$ scenario with $\mu=-700 \mathrm{GeV}$. The values of the mass of the light $\mathcal{C P}$-even Higgs boson, $M_{h}$, are indicated by contour lines. The dark shaded (blue) region corresponds to the parameter region that is excluded by the LEP Higgs searches in the channel $e^{+} e^{-} \rightarrow Z^{*} \rightarrow Z h, H[51,52]$. Lower plot: Contours for the ratio of $A$ signal events in the MSSM over the SM case (with $\left.M_{H^{\mathrm{SM}}}=M_{A}\right)$ for the same parameters. 
such high values of the instantaneous luminosity, in particular the pile-up effect (see Sect. 5), are currently unclear and need further dedicated experimental studies.

Our current tentative conclusion is that the prospects for the observation of the $\mathcal{C} \mathcal{P}$ odd Higgs boson in diffractive processes at the LHC look borderline. Detailed experimental simulation studies would be helpful in order to arrive at a more definite conclusion on the prospects for $A$-boson production in this channel.

\section{Conclusions}

We have analysed in this paper the prospects for probing the Higgs sector of the MSSM with central exclusive Higgs-boson production processes at the LHC, utilising forward proton detectors installed at $220 \mathrm{~m}$ and $420 \mathrm{~m}$ distance around ATLAS and CMS. We have studied CED production of the neutral $\mathcal{C} \mathcal{P}$-even Higgs bosons $h$ and $H$ and their decays into bottom quarks, $\tau$ leptons and $W$ bosons, accounting in each case for the relevant background processes. The experimental efficiencies for the various CED channels are based on existing dedicated studies. The impact of pile-up backgrounds, which are important in particular at high instantaneous LHC luminosity, has been discussed, and various approaches for reducing this background source have been summarised. In order to illustrate the physics potential of the CED processes, four luminosity scenarios have been investigated, corresponding to different assumptions on the achievable overall exerimental efficiencies and the integrated luminosity that can be utilised for the CED channels.

A striking feature of CED Higgs-boson production is that this channel provides good prospects for detecting Higgs-boson decays into bottom quarks, $\tau$ leptons and $W$ bosons. Although the decay into bottom quarks is the dominant decay mode for a light SM-like Higgs boson, this decay channel is very difficult to access in the conventional search channels at the LHC. In the MSSM the $b \bar{b}$ and $\tau^{+} \tau^{-}$decay channels are of particular importance, since they are in general dominant even for heavy MSSM Higgs bosons, whereas a SM Higgs boson of the same mass would have a negligible branching ratio into $b \bar{b}$ and $\tau^{+} \tau^{-}$. It should be noted that heavy Higgs bosons that decouple from gauge bosons and therefore predominantly decay into heavy SM fermions are a quite generic feature of extended Higgs-boson sectors.

We have analysed the $5 \sigma$ discovery contours and contours of $3 \sigma$ statistical significances for the CED channels with $h, H \rightarrow b \bar{b}$ and $h, H \rightarrow \tau^{+} \tau^{-}$in the $M_{A}$-tan $\beta$ parameter plane of the MSSM for various benchmark settings of the other parameters. Concerning the search for the light $\mathcal{C P}$-even Higgs boson, $h$, we find that for the (most optimistic) "600 $\mathrm{fb}^{-1} \mathrm{eff} \times 2$ " scenario the whole $M_{A}-\tan \beta$ plane of the MSSM, with the exception of a small parameter region for small $M_{A}$, can be covered with the CED process at the $3 \sigma$ level. The case of a light SM-like Higgs can also be covered at the $3 \sigma$ level in this luminosity scenario. Thus, if the CED channel can be utilised at high instantaneous luminosity (which requires in particular that pile-up background is brought under control) it can contribute very important information on the Higgs sector of the MSSM. Besides giving access to the bottom Yukawa coupling, which is a crucial input for determining all other Higgs-boson couplings, observation of a Higgs boson in CED production with subsequent decay into bottom quarks would provide information on the $\mathcal{C P}$ quantum numbers of the new state, yield an (additional) precise mass measurement, and may even allow a direct measurement of the Higgs-boson width. We have furthermore 
shown that even if only an integrated luminosity of $60 \mathrm{fb}^{-1}$ can be utilised for the CED production process, the MSSM parameter region with large $\tan \beta$ and relatively small $M_{A}$ can still be probed. For the $h \rightarrow \tau^{+} \tau^{-}$channel we find a slightly weaker coverage compared to the $h \rightarrow b \bar{b}$ channel, based however on conservatively assuming the same selection efficiencies for this channel as for the $h \rightarrow b \bar{b}$ channel. An improved selection procedure could yield a significant gain for the $\tau^{+} \tau^{-}$channel.

We have shown that CED production of the heavier $\mathcal{C} \mathcal{P}$-even Higgs boson of the MSSM with subsequent decay into bottom quarks provides a unique opportunity for accessing its bottom Yukawa coupling in a mass range where for a SM Higgs boson the decay rate into bottom quarks would be negligibly small. In the " $600 \mathrm{fb}^{-1}$ eff $\times 2$ " scenario the discovery of a heavy $\mathcal{C} \mathcal{P}$-even Higgs boson with a mass of about $140 \mathrm{GeV}$ will be possible for all values of $\tan \beta$. This is of particular interest in view of the "wedge region" left uncovered by the conventional search channels for heavy MSSM Higgs bosons. With an effective integrated luminosity of only " $60 \mathrm{fb}^{-1} \mathrm{eff} \times 2$ ", Higgs masses of up to $M_{H} \approx 200 \mathrm{GeV}$ can be probed in the high-tan $\beta$ region at the $3 \sigma$ level. If the bottom Yukawa coupling is enhanced by higher-order corrections, this sensitivity extends beyond $M_{H}=250 \mathrm{GeV}$. For the $H \rightarrow \tau^{+} \tau^{-}$channel, as a consequence of the reduced branching ratio, an effective integrated luminosity of at least $600 \mathrm{fb}^{-1}$ will be necessary to probe significant parts of the MSSM parameter space.

We have furthermore analysed the channels $h, H \rightarrow W W^{(*)}$ in CED production and compared them with the SM case. Since an enhancement of the bottom Yukawa coupling happens at the expense of the branching ratio into $W$ bosons, the $h, H \rightarrow W W^{(*)}$ channel is less favourable compared to the SM case in significant parts of the MSSM parameter space. However, the opposite effect is also possible, giving rise to an enhancement of the CED Higgsboson production with subsequent decay into $W$ bosons compared to the SM case. We have shown that for $140 \mathrm{GeV} \lesssim M_{A} \lesssim 170 \mathrm{GeV}$ and intermediate $\tan \beta$ an enhancement of the MSSM rate of the CED production with $h \rightarrow W W^{(*)}$ of up to a factor of four is possible compared to the SM case. Since the irreducible background to this channel has not been fully investigated yet, we have not presented $5 \sigma$ discovery regions for this channel. Clearly, more detailed experimental studies of the $W W$ modes would be very desirable to assess the physics potential of this interesting channel.

We have furthermore discussed the prospects for identifying the $\mathcal{C P}$-odd Higgs boson, $A$, in diffractive processes at the LHC. Since the CED production of the $\mathcal{C P}$-odd Higgs boson is less promising than production of the $\mathcal{C P}$-even state because of a strong suppression of this mode caused by the $P$-even selection rule, we have investigated $A$ boson production in a less exclusive reaction. The experimental analysis of the double diffractive dissociation of the incoming protons in the presence of severe QCD backgrounds appears to be challenging at the present time. However, further detailed experimental and theoretical studies will be required to reach a firm conclusion on the prospects for this channel.

For clarity of presentation, the analyses in this paper have been performed in the $\mathcal{C P}$ conserving MSSM, i.e. in terms of the $\mathcal{C P}$ eigenstates $h, H, A$. Our analysis could easily be extended to the case where $\mathcal{C P}$-violating complex phases are present, giving rise to a mixing between the three neutral Higgs bosons. In fact, the CED production channels may provide crucial information on the $\mathcal{C P}$ properties of Higgs-like states detected at the LHC. 


\section{Acknowledgements}

We thank Michele Arneodo, Brian Cox, Albert De Roeck, Nigel Glover, Monika Grothe, Alan Martin, Alexandre Nikitenko, Risto Orava and Andrew Pilkington for useful discussions. MGR thanks the IPPP at the University of Durham for hospitality. This work was supported by INTAS grant 05-103-7515, by grant RFBR 07-02-00023, by the Federal Program of Russian Ministry of Industry, Science and Technology RSGSS-5788.2006.02, by the Ministry of Education of the Czech Republic under the project LC527 and by Interuniversity Attraction Poles Programme - Belgian Science Policy. The work of SH was partially supported by CICYT (grant FPA2006-02315). This work is also supported in part by the European Community's Marie-Curie Research Training Network under contract MRTN-CT2006-035505 'Tools and Precision Calculations for Physics Discoveries at Colliders'.

\section{References}

[1] ATLAS Collaboration, Detector and Physics Performance Technical Design Report, CERN/LHCC/99-15 (1999), see: atlasinfo.cern.ch/Atlas/GROUPS/PHYSICS/TDR/access . html .

[2] M. Schumacher, Czech. J. Phys. 54 (2004) A103; hep-ph/0410112.

[3] S. Abdullin et al., Eur. Phys. J. C 39S2 (2005) 41.

[4] CMS Collaboration, Physics Technical Design Report, Volume 2. CERN/LHCC 2006021, see: cmsdoc.cern.ch/cms/cpt/tdr/.

[5] H. Nilles, Phys. Rept. 110 (1984) 1;

H. Haber and G. Kane, Phys. Rept. 117 (1985) 75;

R. Barbieri, Riv. Nuovo Cim. 11 (1988) 1.

[6] M. Carena and H. Haber, Prog. Part. Nucl. Phys. 50 (2003) 63, hep-ph/0208209;

S. Heinemeyer, Int. J. Mod. Phys. A 21 (2006) 2659, hep-ph/0407244;

S. Heinemeyer, W. Hollik and G. Weiglein, Phys. Rept. 425 (2006) 265, hep-ph/0412214;

A. Djouadi, hep-ph/0503172; hep-ph/0503173.

[7] J. Aguilar-Saavedra et al., TESLA TDR Part 3: "Physics at an $e^{+} e^{-}$Linear Collider," hep-ph/0106315, see: tesla.desy.de/tdr/ .

[8] T. Abe et al. [American Linear Collider Working Group Collaboration], Resource book for Snowmass 2001, hep-ex/0106056.

[9] K. Abe et al. [ACFA Linear Collider Working Group Collaboration], hep-ph/0109166.

[10] S. Heinemeyer et al., hep-ph/0511332.

[11] D. Zeppenfeld, R. Kinnunen, A. Nikitenko and E. Richter-Was, Phys. Rev. D 62 (2000) 013009, hep-ph/0002036;

A. Belyaev and L. Reina, JHEP 0208, 041 (2002), hep-ph/0205270. 
[12] M. Dührssen, ATL-PHYS-2003-030, available from http://cdsweb.cern.ch .

[13] M. Dührssen, S. Heinemeyer, H. Logan, D. Rainwater, G. Weiglein and D. Zeppenfeld, Phys. Rev. D 70 (2004) 113009, hep-ph/0406323; hep-ph/0407190.

[14] T. Plehn, D. Rainwater and D. Zeppenfeld, Phys. Rev. Lett. 88 (2002) 051801, hep-ph/0105325;

V. Hankele, G. Klamke, D. Zeppenfeld and T. Figy, Phys. Rev. D 74 (2006) 095001, hep-ph/0609075;

G. Klamke and D. Zeppenfeld, JHEP 0704 (2007) 052, hep-ph/0703202;

C. Ruwiedel, M. Schumacher and N. Wermes, Eur. Phys. J. C 51 (2007) 385.

[15] V.A. Khoze, A.D. Martin and M. Ryskin, Eur. Phys. J. C 14 (2000) 525, hep-ph/0002072.

[16] V.A. Khoze, A.D. Martin and M. Ryskin, Eur. Phys. J. C 23 (2002) 311, hep-ph/0111078.

[17] A. De Roeck, V.A. Khoze, A.D. Martin, R. Orava and M. Ryskin, Eur. Phys. J. C 25 (2002) 391, hep-ph/0207042.

[18] B. Cox, AIP Conf. Proc. 753 (2005) 103, hep-ph/0409144.

[19] J. Ellis, J. Lee and A. Pilaftsis, Phys. Rev. D 70 (2004) 075010, hep-ph/0404167; Phys. Rev. D 71 (2005) 075007, hep-ph/0502251.

[20] M. Albrow et al., CERN-LHCC-2005-025.

[21] V.A. Khoze, A. Kaidalov, A.D. Martin, M. Ryskin and W.J. Stirling, published in Gribov memorial volume 129-144, Budapest 2005, hep-ph/0507040;

A.D. Martin, V.A. Khoze and M. Ryskin, hep-ph/0507305.

[22] M. Boonekamp, R. Peschanski and C. Royon, Phys. Lett. B 598 (2004) 243, hep-ph/0406061; and references therein.

[23] A. Kisselev, V. Petrov and R. Ryutin, Phys. Lett. B 630 (2005) 100, hep-ph/0506034; and references therein.

[24] M. Albrow and A. Rostovtsev, hep-ph/0009336.

[25] V. Berardi et al. [TOTEM Collaboration], TDR, CERN-LHCC-2004-002, TOTEMTDR-001, January 2004.

[26] CMS and TOTEM diffractive and forward physics working group, CERN/LHCC 2006039/G-124, CMS Note 2007/002, TOTEM Note 06-5, December 2006.

[27] RP220 project at ATLAS, see: cern.ch/project-rp220 .

[28] B. Cox, F. Loebinger and A. Pilkington, arXiv:0709.3035 [hep-ph]. 
[29] For a recent review see: J. Forshaw, hep-ph/0508274, published in the proceedings of the workshop HERA and the LHC: A Workshop on the Implications of HERA for LHC Physics, hep-ph/0601013, p. 466.

[30] V.A. Khoze, A.D. Martin and M. Ryskin, in Proc. of 8th Int. Workshop on Deep Inelastic Scattering and QCD (DIS2000), Liverpool, eds. J. Gracey and T. Greenshaw (World Scientific, 2001), p.592, hep-ph/0006005.

[31] V.A. Khoze, A.D. Martin and M. Ryskin, Eur. Phys. J. C 19 (2001) 477 [Erratum-ibid. C 20 (2001) 599], hep-ph/0011393.

[32] J. Kalliopuska et al., HIP-2003-11/EXP;

published in the proceedings of the workshop HERA and the LHC: A Workshop on the Implications of HERA for LHC Physics, hep-ph/0601013, p. 448.

[33] A. Kaidalov, V.A. Khoze, A.D. Martin and M. Ryskin, Eur. Phys. J. C 33 (2004) 261, hep-ph/0311023.

[34] M. Boonekamp, J. Cammin, S. Lavignac, R. Peschanski and C. Royon, Phys. Rev. D 73 (2006) 115011, hep-ph/0506275.

[35] V.A. Khoze, A.D. Martin and M. Ryskin, Eur. Phys. J. C 34 (2004) 327, hep-ph/0401078.

[36] S. Gennai, S. Heinemeyer, A. Kalinowski, R. Kinnunen, S. Lehti, A. Nikitenko and G. Weiglein, arXiv:0704.0619 [hep-ph].

[37] S. Heinemeyer, W. Hollik and G. Weiglein, Comp. Phys. Commun. 1242000 76, hep-ph/9812320. The code is accessible via www.feynhiggs.de .

[38] G. Degrassi, S. Heinemeyer, W. Hollik, P. Slavich and G. Weiglein, Eur. Phys. J. C 28 (2003) 133, hep-ph/0212020.

[39] S. Heinemeyer, W. Hollik and G. Weiglein, Eur. Phys. J. C 9 (1999) 343, hep-ph/9812472.

[40] M. Frank, T. Hahn, S. Heinemeyer, W. Hollik, H. Rzehak and G. Weiglein, JHEP 0702 (2007) 047, hep-ph/0611326.

[41] R. Hempfling, Phys. Rev. D 49 (1994) 6168;

L. Hall, R. Rattazzi and U. Sarid, Phys. Rev. D 50 (1994) 7048, hep-ph/9306309;

M. Carena, M. Olechowski, S. Pokorski and C. Wagner, Nucl. Phys. B 426 (1994) 269, hep-ph/9402253.

[42] M. Carena, D. Garcia, U. Nierste and C. Wagner, Nucl. Phys. B 577 (2000) 577, hep-ph/9912516.

[43] H. Eberl, K. Hidaka, S. Kraml, W. Majerotto and Y. Yamada, Phys. Rev. D 62 (2000) 055006, hep-ph/9912463. 
[44] J. Guasch, P. Häfliger and M. Spira, Phys. Rev. D 68 (2003) 115001, hep-ph/0305101.

[45] A. Brignole, G. Degrassi, P. Slavich and F. Zwirner, Nucl. Phys. B 643 (2002) 79, hep-ph/0206101.

[46] G. Degrassi, A. Dedes and P. Slavich, Nucl. Phys. B 672 (2003) 144, hep-ph/0305127.

[47] S. Heinemeyer, W. Hollik, H. Rzehak and G. Weiglein, Eur. Phys. J. C 39 (2005) 465, hep-ph/0411114; hep-ph/0506254.

[48] S. Heinemeyer, W. Hollik and G. Weiglein, Eur. Phys. J. C 16 (2000) 139, hep-ph/0003022.

[49] M. Carena, S. Heinemeyer, C. Wagner and G. Weiglein, Eur. Phys. J. C 26 (2003) 601, hep-ph/0202167.

[50] M. Carena, S. Heinemeyer, C. Wagner and G. Weiglein, Eur. Phys. J. C 45 (2006) 797, hep-ph/0511023.

[51] G. Abbiendi et al. [ALEPH, DELPHI, L3, OPAL Collaborations and LEP Working Group for Higgs boson searches], Phys. Lett. B 565 (2003) 61, hep-ex/0306033.

[52] S. Schael et al. [ALEPH, DELPHI, L3, OPAL Collaborations and LEP Working Group for Higgs boson searches], Eur. Phys. J. C 47 (2006) 547, hep-ex/0602042.

[53] V. Abazov et al. [D0 Collaboration], Phys. Rev. Lett. 95 (2005) 151801, hep-ex/0504018; Phys. Rev. Lett. 97 (2006) 121802, hep-ex/0605009.

[54] [D0 Collaboration], D0 Note 5331-CONF.

[55] A. Abulencia et al. [CDF Collaboration], Phys. Rev. Lett. 96 (2006) 011802, hep-ex/0508051.

[56] [CDF Collaboration], CDF note 8676.

[57] [CDF Collaboration], Phys. Rev. Lett. 96 (2006) 042003, hep-ex/0510065;

R. Eusebi, Ph.d. thesis: "Search for charged Higgs in $t \bar{t}$ decay products from protonantiproton collisions at $\sqrt{s}=1.96 \mathrm{TeV}$ ", University of Rochester, 2005 .

[58] S. Heinemeyer, W. Hollik and G. Weiglein, JHEP 0006 (2000) 009, hep-ph/9909540.

[59] Tevatron Electroweak Working Group, hep-ex/0703034.

[60] V.A. Khoze, A.D. Martin and M. Ryskin, Eur. Phys. J. C 18 (2000) 167, hep-ph/0007359.

[61] For a recent review see: E. Gotsman, E. Levin, U. Maor, E. Naftali and A. Prygarin, hep-ph/0511060;

published in the proceedings of the workshop HERA and the LHC: A Workshop on the Implications of HERA for LHC Physics, hep-ph/0601012, p. 221. 
[62] A. Kaidalov, V.A. Khoze, A.D. Martin and M. Ryskin, Eur. Phys. J. C 31 (2003) 387, hep-ph/0307064.

[63] J. Monk and A. Pilkington, Comput. Phys. Commun. 175 (2006) 232, hep-ph/0502077.

[64] M. Boonekamp, C. Hogg, J. Monk, A. Pilkington and M. Tasevsky, published in the proceedings of the workshop HERA and the LHC: A Workshop on the Implications of HERA for LHC Physics, hep-ph/0601013, p. 482.

[65] V.A. Khoze, A.D. Martin, M. Ryskin and W.J. Stirling, Eur. Phys. J. C 38 (2005) 475, hep-ph/0409037.

[66] K. Goulianos, FERMILAB-CONF-06-429-E, Nov 2006, PoS D IFF2006 (2006) 016; K. Terashi [CDF and D0 Collaboration], hep-ex/0605084.

[67] V.A. Khoze, M. Ryskin and W.J. Stirling, Eur. Phys. J. C 48 (2006) 797, hep-ph/0607134.

[68] V. Fadin, V.A. Khoze and A.D. Martin, Phys. Rev. D 56 (1997) 484, hep-ph/9703402.

[69] D. Borden, V.A. Khoze, W.J. Stirling and J. Ohnemus, Phys. Rev. D 50 (1994) 4499, hep-ph/9405401.

[70] V.A. Khoze, A.D. Martin and M. Ryskin, Phys. Lett. B 650 (2007) 41, hep-ph/0702213.

[71] V.A. Khoze, A.D. Martin and M. Ryskin, Eur. Phys. J. C 26 (2002) 229, hep-ph/0207313.

[72] A.D. Martin, M. Ryskin and G. Watt, Phys. Lett. B 644 (2007) 131, hep-ph/0609273.

[73] B. Cox and J. Forshaw, Comput. Phys. Commun. 144 (2002) 104, hep-ph/0010303.

[74] A. Aktas et al. [H1 Collaboration], Eur. Phys. J. C 48 (2006) 715, hep-ex/0606004.

[75] V.A. Khoze, A.D. Martin and M. Ryskin, Eur. Phys. J. C 24 (2002) 459, hep-ph/0201301.

[76] D. Cavalli et al., ATLAS Internal note, PHYS-NO-051, 1994;

R. Kinnunen and A. Nikitenko, CMS Note 1997/002.

[77] B. Cox et al., Eur. Phys. J. C 45 (2006) 401, hep-ph/0505240.

[78] V.A. Khoze, M. Ryskin and W.J. Stirling, Eur. Phys. J. C 44 (2005) 227, hep-ph/0504131.

[79] M. Albrow et al., CERN-LHCC-2005-025, June 2005.

[80] M. Grothe et al., CERN-CMS-NOTE-2006-054, 2006. 
[81] I. Narsky, Nucl.Instrum.Meth. A 450 (2000) 444, hep-ex/9904025;

G. Quast, CMS Physics Analysis Days, May 9, 2005;

S. Bityukov et al., in the Conference Proceedings of PHYSTAT2005: Statistical Problems in Particle Physics, Astrophysics, and Cosmology, Editors: L. Lyons, M. Unel, Imperial College Press, 2006, p. 106.

[82] M. Grothe, private communication.

[83] FP420 TDR, see: www.fp420.com .

[84] O. Brüning et al., CERN-LHC-PROJECT-REPORT-626; F. Gianotti et al., CERNTH/2002-078, hep-ph/0204087.

[85] V.A. Khoze, A.D. Martin and M. Ryskin, Phys. Lett. B 643 (2006) 93, hep-ph/0609312.

[86] R. Engel, Z. Phys. C 66 (1995) 203;

R. Engel and J. Ranft, Phys. Rev. D 54 (1996) 4244, hep-ph/9509373.

[87] A. Pilkington, M. Tasevsky, talks at the HERA-LHC workshop, DESY Hamburg, March 12-16, 2007.

[88] [CDF collaboration], arXiv:0707.2374 [hep-ex].

[89] V.A. Khoze, M. Ryskin, W.J. Stirling and P. Williams, Eur. Phys. J. C 26 (2003) 429, hep-ph/0207365. 